\title{
Riesz Transform Characterizations of Musielak-Orlicz-Hardy Spaces
}

\author{
Jun Cao, Der-Chen Chang, Dachun Yang* and Sibei Yang
}

\begin{abstract}
Let $\varphi$ be a Musielak-Orlicz function satisfying that, for any $(x, t) \in$ $\mathbb{R}^{n} \times(0, \infty), \varphi(\cdot, t)$ belongs to the Muckenhoupt weight class $A_{\infty}\left(\mathbb{R}^{n}\right)$ with the critical weight exponent $q(\varphi) \in[1, \infty)$ and $\varphi(x, \cdot)$ is an Orlicz function with $0<i(\varphi) \leq I(\varphi) \leq 1$ which are, respectively, its critical lower type and upper type. In this article, the authors establish the Riesz transform characterizations of the Musielak-Orlicz-Hardy spaces $H_{\varphi}\left(\mathbb{R}^{n}\right)$ which are generalizations of weighted Hardy spaces and Orlicz-Hardy spaces. Precisely, the authors characterize $H_{\varphi}\left(\mathbb{R}^{n}\right)$ via all the first order Riesz transforms when $\frac{i(\varphi)}{q(\varphi)}>\frac{n-1}{n}$, and via all the Riesz transforms with the order not more than $m \in \mathbb{N}$ when $\frac{i(\varphi)}{q(\varphi)}>\frac{n-1}{n+m-1}$. Moreover, the authors also establish the Riesz transform characterizations of $H_{\varphi}\left(\mathbb{R}^{n}\right)$, respectively, by means of the higher order Riesz transforms defined via the homogenous harmonic polynomials or the odd order Riesz transforms. Even if when $\varphi(x, t):=t w(x)$ for all $x \in \mathbb{R}^{n}$ and $t \in[0, \infty)$, these results also widen the range of weights in the known Riesz characterization of the classical weighted Hardy space $H_{w}^{1}\left(\mathbb{R}^{n}\right)$ obtained by R. L. Wheeden from $w \in A_{1}\left(\mathbb{R}^{n}\right)$ into $w \in A_{\infty}\left(\mathbb{R}^{n}\right)$ with the sharp range $q(w) \in\left[1, \frac{n}{n-1}\right)$, where $q(w)$ denotes the critical index of the weight $w$.
\end{abstract}

\section{Introduction}

Denote by $\mathcal{S}\left(\mathbb{R}^{n}\right)$ the space of all Schwartz functions on $\mathbb{R}^{n}$. For $j \in\{1, \ldots, n\}$, $f \in \mathcal{S}\left(\mathbb{R}^{n}\right)$ and $x \in \mathbb{R}^{n}$, the $j$-th Riesz transform of $f$ is usually defined by

$$
R_{j}(f)(x):=\lim _{\epsilon \rightarrow 0^{+}} C_{(n)} \int_{\left\{y \in \mathbb{R}^{n}:|y|>\epsilon\right\}} \frac{y_{j}}{|y|^{n+1}} f(x-y) d y,
$$

here and hereafter, $\epsilon \rightarrow 0^{+}$means that $\epsilon>0$ and $\epsilon \rightarrow 0, C_{(n)}:=\frac{\Gamma((n+1) / 2)}{\pi^{(n+1) / 2}}$ and $\Gamma$ denotes the Gamma function. As a natural generalization of the Hilbert transform to the Euclidean space of higher dimension, Riesz transforms may be the most typical examples of CalderónZygmund operators which have been extensively studied by many mathematicians (see, for example, [31, 32, 13] and their references).

2010 Mathematics Subject Classification. Primary: 47B06; Secondary: 42B20, 42B30, 42B35, 46 E30.

Key words and phrases. Riesz transform, harmonic function, Cauchy-Riemann equation, MusielakOrlicz-Hardy space.

Jun Cao is supported by the Fundamental Research Funds for the Central Universities (Grant No. 2012YBXS16). Der-Chen Chang is partially supported by an NSF grant DMS-1203845 and Hong Kong RGC competitive earmarked research grant \#601410. Dachun Yang is supported by the National Natural Science Foundation of China (Grant Nos. 11171027 and 11361020) and the Specialized Research Fund for the Doctoral Program of Higher Education of China (Grant No. 20120003110003).

*Corresponding author 
While most literatures on Riesz transforms focus on their boundedness on various function spaces, the main purpose of this article is to establish Riesz transform characterizations of some Hardy spaces of Musielak-Orlicz type. This research originates from Fefferman-Stein's 1972 celebrating seminal paper [9] and was then extended by Wheeden to the weighted Hardy space $H_{w}^{1}\left(\mathbb{R}^{n}\right)$ (see [4]). It is known that, when establishing Riesz transform characterizations of Hardy spaces $H^{p}\left(\mathbb{R}^{n}\right)$, we need to extend the elements of $H^{p}\left(\mathbb{R}^{n}\right)$ to the upper half space $\mathbb{R}_{+}^{n+1}:=\mathbb{R}^{n} \times(0, \infty)$ via the Poisson integral. This extension in turn has close relationship with the analytical definition of $H^{p}\left(\mathbb{R}^{n}\right)$ which is the key starting point of studying the Hardy space, before people paid attention to the real-variable theory of $H^{p}\left(\mathbb{R}^{n}\right)$ (see $[33,30,34,26,42]$ ). Recall also that the real-variable theory of $H^{p}\left(\mathbb{R}^{n}\right)$ and their weighted versions plays very important roles in analysis such as harmonic analysis and partial differential equations; see, for example, [32, 14, 7].

The Riesz transform characterization of Hardy spaces on $\mathbb{R}^{n}$ is one of the most important and useful real-variable characterizations (see [9, 26, 42, 32, 38]). Indeed it is well known that Riesz transforms have many interesting properties. For example, they are the simplest, non-trivial, "invariant" operators under the acting of the group of rotations in the Euclidean space $\mathbb{R}^{n}$, and they also constitute typical and important examples of Fourier multipliers. Moreover, they can be used to mediate between various combinations of partial derivatives of functions. All these properties make Riesz transforms ubiquitous in mathematics (see [31] for more details on their applications). Recall also that Riesz transforms are not bounded on Lebesgue spaces $L^{p}\left(\mathbb{R}^{n}\right)$ when $p \in(0,1]$. One of the main motivations to introduce the Hardy space $H^{p}\left(\mathbb{R}^{n}\right)$ with $p \in(0,1]$ is to find a suitable substitute of $L^{p}\left(\mathbb{R}^{n}\right)$ when studying the boundedness of some operators.

Denote by $\mathcal{S}^{\prime}\left(\mathbb{R}^{n}\right)$ the dual space of $\mathcal{S}\left(\mathbb{R}^{n}\right)$ (namely, the space of all tempered distributions). Let $f \in \mathcal{S}^{\prime}\left(\mathbb{R}^{n}\right)$. Recall that a distribution $f \in \mathcal{S}^{\prime}\left(\mathbb{R}^{n}\right)$ is called a distribution restricted at infinity, if there exists a positive number $r$ sufficiently large such that, for all $\phi \in \mathcal{S}\left(\mathbb{R}^{n}\right), f * \phi \in L^{r}\left(\mathbb{R}^{n}\right)$. Fefferman and Stein [9] proved the following important result (see also [32, p. 123, Proposition 3] for a more detailed description).

Theorem $1.1([9])$. Let $p \in\left(\frac{n-1}{n}, \infty\right), \phi \in \mathcal{S}\left(\mathbb{R}^{n}\right)$ satisfy $\int_{\mathbb{R}^{n}} \phi(x) d x=1$ and $f$ be a distribution restricted at infinity. Then $f \in H^{p}\left(\mathbb{R}^{n}\right)$ if and only if there exists a positive constant $A$ such that, for all $\epsilon \in(0, \infty)$ and $j \in\{1, \ldots, n\}, f * \phi_{\epsilon}, R_{j}(f) * \phi_{\epsilon} \in L^{p}\left(\mathbb{R}^{n}\right)$ and

$$
\left\|f * \phi_{\epsilon}\right\|_{L^{p}\left(\mathbb{R}^{n}\right)}+\sum_{j=1}^{n}\left\|R_{j}(f) * \phi_{\epsilon}\right\|_{L^{p}\left(\mathbb{R}^{n}\right)} \leq A,
$$

where, for all $x \in \mathbb{R}^{n}, \phi_{\epsilon}(x):=\frac{1}{\epsilon^{n}} \phi\left(\frac{x}{\epsilon}\right)$. Moreover, there exists a positive constant $C$, independent of $f$ and $\epsilon$, such that

$$
\frac{1}{C}\|f\|_{H^{p}\left(\mathbb{R}^{n}\right)} \leq A \leq C\|f\|_{H^{p}\left(\mathbb{R}^{n}\right)} .
$$

It is known that, for $p \in\left(0, \frac{n-1}{n}\right], H^{p}\left(\mathbb{R}^{n}\right)$ can be characterized no longer by first order Riesz transforms but by higher order Riesz transforms (see [9, p. 168] or Theorem 1.7 below for more details). 
In this article, we establish the Riesz transform characterization of the Musielak-OrliczHardy space $H_{\varphi}\left(\mathbb{R}^{n}\right)$ which is introduced by $\mathrm{Ky}\left[23\right.$. It is known that the space $H_{\varphi}\left(\mathbb{R}^{n}\right)$ is a generalization of the Orlicz-Hardy space introduced by Strömberg [36] and Janson [19], and the weighted Hardy space $H_{w}^{p}\left(\mathbb{R}^{n}\right)$ for $w \in A_{\infty}\left(\mathbb{R}^{n}\right)$ and $p \in(0,1]$, introduced by García-Cuerva [1] and Strömberg-Torchinsky [37. Here, $A_{q}\left(\mathbb{R}^{n}\right)$ with $q \in[1, \infty]$ denotes the class of Muckenhoupt weights (see, for example, [11, 12, 13] for their definitions and properties). Moreover, the space $H_{\varphi}\left(\mathbb{R}^{n}\right)$ also has already found many applications in analysis (see, for example, [3, 4, 17, 22, 23] and their references).

Recall that, in [41], Wheeden characterized the weighted Hardy space $H_{w}^{1}\left(\mathbb{R}^{n}\right)$ via first order Riesz transforms when $w \in A_{1}\left(\mathbb{R}^{n}\right)$. Our results extend the corresponding results of [9, 41] essentially; see Remark 1.6 below for more details.

In order to state the main results of this article, let us recall some necessary definitions and notation.

Let $\varphi$ be a nonnegative function on $\mathbb{R}^{n} \times[0, \infty)$. The function $\varphi$ is called a MusielakOrlicz function, if, for any $x \in \mathbb{R}^{n}, \varphi(x, \cdot)$ is an Orlicz function on $[0, \infty)$ and, for any $t \in$ $[0, \infty), \varphi(\cdot, t)$ is measurable on $\mathbb{R}^{n}$. Here a function $\Phi:[0, \infty) \rightarrow[0, \infty)$ is called an Orlicz function, if it is nondecreasing, $\Phi(0)=0, \Phi(t)>0$ for $t \in(0, \infty)$ and $\lim _{t \rightarrow \infty} \Phi(t)=\infty$ (see, for example, [27]). Remark that, unlike the usual case, such a $\Phi$ may not be convex.

For an Orlicz function $\Phi$, the most useful tool to study its growth property may be the upper and the lower types of $\Phi$. More precisely, for $p \in(0, \infty)$, a function $\Phi$ is said to be of upper (resp. lower) type $p$, if there exists a positive constant $C$ such that, for all $s \in[1, \infty)$ (resp. $s \in[0,1])$ and $t \in[0, \infty)$,

$$
\Phi(s t) \leq C s^{p} \Phi(t) .
$$

Let $\varphi$ be a Musielak-Orlicz function. The Musielak-Orlicz space $L^{\varphi}\left(\mathbb{R}^{n}\right)$, which was first introduced by Musielak [27], is defined to be the set of all measurable functions $f$ such that $\int_{\mathbb{R}^{n}} \varphi(x,|f(x)|) d x<\infty$ with the Luxembourg-Nakano (quasi-)norm:

$$
\|f\|_{L^{\varphi}\left(\mathbb{R}^{n}\right)}:=\inf \left\{\lambda \in(0, \infty): \int_{\mathbb{R}^{n}} \varphi\left(x, \frac{|f(x)|}{\lambda}\right) d x \leq 1\right\} .
$$

We also need the following notion of Muckenhoupt weight classes from [25]. For $q \in$ $(1, \infty)$, a nonnegative locally integrable function $w$ on $\mathbb{R}^{n}$ is said to belong to $A_{q}\left(\mathbb{R}^{n}\right)$, if, for all balls $B \subset \mathbb{R}^{n}$,

$$
\left\{\frac{1}{|B|} \int_{B} w(x) d x\right\}\left\{\frac{1}{|B|} \int_{B}[w(x)]^{1-q^{\prime}} d x\right\}^{q-1} \leq[w]_{A_{q}\left(\mathbb{R}^{n}\right)}<\infty,
$$

here and hereafter, $q^{\prime}:=\frac{q}{q-1}$ denotes the conjugate exponent of $q$. Moreover, the nonnegative locally integrable function $w$ is said to belong to $A_{1}\left(\mathbb{R}^{n}\right)$, if, for all balls $B \subset \mathbb{R}^{n}$,

$$
\left\{\frac{1}{|B|} \int_{B} w(x) d x\right\}\left\{\underset{y \in B}{\operatorname{ess} \sup }[w(y)]^{-1}\right\} \leq[w]_{A_{1}\left(\mathbb{R}^{n}\right)}<\infty .
$$

Let $A_{\infty}\left(\mathbb{R}^{n}\right):=\cup_{q \in[1, \infty)} A_{q}\left(\mathbb{R}^{n}\right)$. Moreover, throughout the whole article, we always assume that the Musielak-Orlicz functions satisfy the following growth assumptions (see [23, Definition 2.1]). 
Assumption $(\varphi)$. Let $\varphi: \mathbb{R}^{n} \times[0, \infty) \rightarrow[0, \infty)$ be a Musielak-Orlicz function satisfying the following two conditions:

(i) for any $t \in(0, \infty), \varphi(\cdot, t) \in A_{\infty}\left(\mathbb{R}^{n}\right)$;

(ii) there exists $p \in(0,1]$ such that, for every $x \in \mathbb{R}^{n}, \varphi(x, \cdot)$ is of upper type 1 and of lower type $p$.

Notice that there exist many examples of functions satisfying Assumption $(\varphi)$. For example, for all $(x, t) \in \mathbb{R}^{n} \times[0, \infty), \varphi(x, t):=\omega(x) \Phi(t)$ satisfies Assumption $(\varphi)$ if $\omega \in A_{\infty}\left(\mathbb{R}^{n}\right)$ and $\Phi$ is an Orlicz function of lower type $p$ for some $p \in(0,1]$ and upper type 1. A typical example of such an Orlicz function $\Phi$ is $\Phi(t):=t^{p}$, with $p \in(0,1]$, for all $t \in[0, \infty)$; see, for example, [17, 22, 23] for more examples. Another typical example of functions satisfying Assumption $(\varphi)$ is $\varphi(x, t):=\frac{t^{\alpha}}{[\ln (e+|x|)]^{\beta}+[\ln (e+t)]^{\gamma}}$ for all $x \in \mathbb{R}^{n}$ and $t \in[0, \infty)$ with any $\alpha \in(0,1]$ and $\beta, \gamma \in[0, \infty)$ (see [23] for further details).

For a Musielak-Orlicz function $\varphi$ satisfying Assumption $(\varphi)$, the following critical indices are useful. Let

$$
\begin{array}{r}
I(\varphi):=\inf \left\{p \in(0, \infty): \text { for any } x \in \mathbb{R}^{n}, \varphi(x, \cdot) \text { is of upper type } p\right. \\
\text { with } C \text { as in (1.2) independent of } x\}, \\
i(\varphi):=\sup \left\{p \in(0, \infty): \text { for any } x \in \mathbb{R}^{n}, \varphi(x, \cdot) \text { is of lower type } p\right. \\
\text { with } C \text { as in (1.2) independent of } x\}
\end{array}
$$

and

$$
\begin{gathered}
q(\varphi):=\inf \left\{q \in[1, \infty): \text { for any } t \in(0, \infty), \varphi(\cdot, t) \in A_{q}\left(\mathbb{R}^{n}\right)\right. \\
\text { with } \left.[\varphi(\cdot, t)]_{A_{q}\left(\mathbb{R}^{n}\right)} \text { independent of } t\right\} .
\end{gathered}
$$

Let $\mathbb{N}:=\{1,2, \ldots\}$ and $\mathbb{Z}_{+}:=\{0\} \cup \mathbb{N}$. For any $\theta:=\left(\theta_{1}, \ldots, \theta_{n}\right) \in \mathbb{Z}_{+}^{n}$, let $|\theta|:=$ $\theta_{1}+\cdots+\theta_{n}$ and $\partial_{x}^{\theta}:=\frac{\partial^{|\theta|}}{\partial x_{1}^{\theta_{1} \ldots \partial x_{n}^{\theta_{n}}}}$. For $m \in \mathbb{N}$, define

$$
\mathcal{S}_{m}\left(\mathbb{R}^{n}\right):=\left\{\phi \in \mathcal{S}\left(\mathbb{R}^{n}\right): \sup _{x \in \mathbb{R}^{n}} \sup _{\beta \in \mathbb{Z}_{+}^{n},|\beta| \leq m+1}(1+|x|)^{(m+2)(n+1)}\left|\partial_{x}^{\beta} \phi(x)\right| \leq 1\right\} .
$$

Then, for all $x \in \mathbb{R}^{n}$ and $f \in \mathcal{S}^{\prime}\left(\mathbb{R}^{n}\right)$, the non-tangential grand maximal function $f_{m}^{*}$ of $f$ is defined by setting,

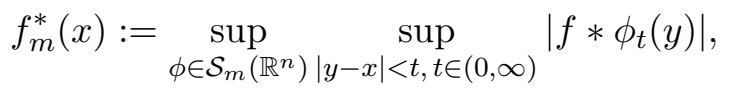

where, for all $t \in(0, \infty), \phi_{t}(\cdot):=t^{-n} \phi(\dot{\bar{t}})$. When $m(\varphi):=\lfloor n[q(\varphi) / i(\varphi)-1]\rfloor$, where $q(\varphi)$ and $i(\varphi)$ are, respectively, as in (1.6) and (1.5), and $\lfloor s\rfloor$ for $s \in \mathbb{R}$ denotes the maximal integer not more than $s$, we denote $f_{m(\varphi)}^{*}$ simply by $f^{*}$.

Ky [23] introduced the following Musielak-Orlicz-Hardy spaces $H_{\varphi}\left(\mathbb{R}^{n}\right)$.

Definition $1.2([23)$. Let $\varphi$ satisfy Assumption $(\varphi)$. The Musielak-Orlicz-Hardy space $H_{\varphi}\left(\mathbb{R}^{n}\right)$ is defined to be the space of all $f \in \mathcal{S}^{\prime}\left(\mathbb{R}^{n}\right)$ such that $f^{*} \in L^{\varphi}\left(\mathbb{R}^{n}\right)$ with the quasi-norm $\|f\|_{H_{\varphi}\left(\mathbb{R}^{n}\right)}:=\left\|f^{*}\right\|_{L^{\varphi}\left(\mathbb{R}^{n}\right)}$. 
Remark 1.3. (i) We point out that, if $\varphi(x, t):=w(x) t^{p}$, with $p \in(0,1]$ and $w \in A_{\infty}\left(\mathbb{R}^{n}\right)$, for all $(x, t) \in \mathbb{R}^{n} \times[0, \infty)$, the Musielak-Orlicz-Hardy space $H_{\varphi}\left(\mathbb{R}^{n}\right)$ coincides with the weighted Hardy space $H_{w}^{p}\left(\mathbb{R}^{n}\right)$ studied in [11, 37]; if $\varphi(x, t):=\Phi(t)$, with $\Phi$ an Orlicz function whose upper type is 1 and lower type $p \in(0,1]$, for all $(x, t) \in \mathbb{R}^{n} \times[0, \infty)$, $H_{\varphi}\left(\mathbb{R}^{n}\right)$ coincides with the Orlicz-Hardy space $H_{\Phi}\left(\mathbb{R}^{n}\right)$ introduced in [19, 36]. Also, the Musielak-Orlicz-Hardy space $H_{\varphi}\left(\mathbb{R}^{n}\right)$ has proved useful in the study of other analysis problems when we take various different Musielak-Orlicz functions $\varphi$ (see, for example, [3, 22, 23]).

(ii) For all $(x, t) \in \mathbb{R}^{n} \times[0, \infty)$, let

$$
\widetilde{\varphi}(x, t):=\int_{0}^{t} \frac{\varphi(x, s)}{s} d s .
$$

It is easy to see that $\widetilde{\varphi}$ is strictly increasing and continuous in $t$. Similar to [40, Proposition $3.1]$, we know that $\widetilde{\varphi}$ inherits the types of $\varphi$ and is equivalent to $\varphi$, which implies that $H_{\varphi}\left(\mathbb{R}^{n}\right)=H_{\widetilde{\varphi}}\left(\mathbb{R}^{n}\right)$ with equivalent quasi-norms. Thus, without loss of generality, in the remainder of this article, we may always assume that $\varphi(x, \cdot)$ for all $x \in \mathbb{R}^{n}$ is strictly increasing and continuous on $[0, \infty)$.

(iii) Let $\overline{H_{\varphi}\left(\mathbb{R}^{n}\right) \cap L^{2}\left(\mathbb{R}^{n}\right)} \|^{\|\cdot\|_{H_{\varphi}\left(\mathbb{R}^{n}\right)}}$ be the completion of the set $H_{\varphi}\left(\mathbb{R}^{n}\right) \cap L^{2}\left(\mathbb{R}^{n}\right)$ under the quasi-norm $\|\cdot\|_{H_{\varphi}\left(\mathbb{R}^{n}\right)}$. From the fact that $H_{\varphi}\left(\mathbb{R}^{n}\right) \cap L^{2}\left(\mathbb{R}^{n}\right)$ is dense in $H_{\varphi}\left(\mathbb{R}^{n}\right)$ which is a simple corollary of [23, Theorem 3.1] in the case $q=\infty$, we immediately deduce that

$$
\overline{H_{\varphi}\left(\mathbb{R}^{n}\right) \cap L^{2}\left(\mathbb{R}^{n}\right)}\|\cdot\|_{H_{\varphi}\left(\mathbb{R}^{n}\right)}=H_{\varphi}\left(\mathbb{R}^{n}\right) .
$$

In order to obtain Riesz transform characterizations of $H_{\varphi}\left(\mathbb{R}^{n}\right)$, we have to overcome some essential difficulties, which have already existed even in the case of weighted Hardy spaces $H_{w}^{p}\left(\mathbb{R}^{n}\right)$, caused by weights. One of the most typical difficulties relies on the fact that, for an arbitrary $f \in H_{\varphi}\left(\mathbb{R}^{n}\right)$, we cannot obtain directly that $f$ is a distribution restricted at infinity as in the unweighted case. To be more precise, let $\phi \in \mathcal{S}\left(\mathbb{R}^{n}\right)$ with $\int_{\mathbb{R}^{n}} \phi(x) d x=1, p \in(0, i(\varphi)), t \in(0, \infty)$ and $x \in \mathbb{R}^{n}$, assume that $\left|f * \phi_{t}(x)\right| \geq 1$; then, following Stein's argument (see [32, pp. 100-101]) and using the lower type $p$ property of $\varphi(\cdot, t)$, we see that

$$
\left|f * \phi_{t}(x)\right|^{p} \lesssim \frac{\int_{B(x, 1)}\left|f * \phi_{t}(x)\right|^{p} \varphi(y, 1) d y}{\int_{B(x, 1)} \varphi(y, 1) d y} \lesssim\|f\|_{H_{\varphi}\left(\mathbb{R}^{n}\right)}^{p} \frac{1}{\int_{B(x, 1)} \varphi(y, 1) d y} .
$$

From this, it follows that, in order to show that $f * \phi_{t} \in L^{\infty}\left(\mathbb{R}^{n}\right)$, we need $\frac{1}{\int_{B(x, 1)} \varphi(y, 1) d y}$ is uniformly bounded in $x$ (see [5, Remark 3.3] for a similar condition in the case of weighted Hardy spaces).

To get rid of this unpleasant and awkward restriction, we aptly adapt a smart and wise strategy that has recently been used in the case of Hardy spaces associated with operators (see, for example, [15, Theorem 5.2] for Riesz transform characterizations of Hardy spaces associated with second order divergence form elliptic operators). Precisely, we first restrict the working space to $H_{\varphi}\left(\mathbb{R}^{n}\right) \cap L^{2}\left(\mathbb{R}^{n}\right)$, in which the Riesz transforms and Poisson integrals are well defined, then we extend the working space by a process of completion via the quasi-norm based on Riesz transforms. In particular, we introduce the following Riesz Musielak-Orlicz-Hardy space. 
Definition 1.4. Let $\varphi$ satisfy Assumption $(\varphi)$. The Riesz Musielak-Orlicz-Hardy space $H_{\varphi, \text { Riesz }}\left(\mathbb{R}^{n}\right)$ is defined to be the completion of the set

$$
\mathbb{H}_{\varphi, \operatorname{Riesz}}\left(\mathbb{R}^{n}\right):=\left\{f \in L^{2}\left(\mathbb{R}^{n}\right):\|f\|_{H_{\varphi, \text { Riesz }}\left(\mathbb{R}^{n}\right)}<\infty\right\}
$$

under the quasi-norm $\|\cdot\|_{H_{\varphi, \text { Riesz }}\left(\mathbb{R}^{n}\right)}$, where, for all $f \in L^{2}\left(\mathbb{R}^{n}\right)$,

$$
\|f\|_{H_{\varphi, \text { Riesz }}\left(\mathbb{R}^{n}\right)}:=\|f\|_{L^{\varphi}\left(\mathbb{R}^{n}\right)}+\sum_{j=1}^{n}\left\|R_{j}(f)\right\|_{L^{\varphi}\left(\mathbb{R}^{n}\right)} .
$$

Now we give out the first main result of this article.

Theorem 1.5. Let $\varphi$ satisfy Assumption $(\varphi)$ and $\frac{i(\varphi)}{q(\varphi)} \in\left(\frac{n-1}{n}, \infty\right)$ with $i(\varphi)$ and $q(\varphi)$ as in (1.5) and (1.6), respectively. Then $H_{\varphi}\left(\mathbb{R}^{n}\right)=H_{\varphi, \text { Riesz }}\left(\mathbb{R}^{n}\right)$ with equivalent quasi-norms.

Remark 1.6. (i) We point out that, if $\varphi(x, t):=t^{p}$, with $p \in\left(\frac{n-1}{n}, 1\right]$, for all $(x, t) \in$ $\mathbb{R}^{n} \times[0, \infty)$, then the difficulty in (1.8) disappear automatically. Thus, in this case, there is no need to use the restriction to $L^{2}\left(\mathbb{R}^{n}\right)$. Observe that, in this case, the range $p \in\left(\frac{n-1}{n}, 1\right]$ in Theorem [1.5 coincides with the range of $p$ in Theorem 1.1 obtained by Fefferman and Stein [9], which is the known best possible. Moreover, compared with Theorem 1.1, an advantage of Theorem 1.5 is that, in Theorem 1.5, we do not assume the a priori assumption that $f$ is a distribution restricted at infinity.

(ii) Recall that Wheeden in [41] characterized $H_{w}^{1}\left(\mathbb{R}^{n}\right)$, with $w \in A_{1}\left(\mathbb{R}^{n}\right)$, by the first order Riesz transforms, which corresponds to the case when $\varphi(x, t):=t w(x)$ for all $x \in \mathbb{R}^{n}$ and $t \in[0, \infty)$ of Theorem 1.5, even in this special case, Theorem 1.5 also widens the range of weights from $w \in A_{1}\left(\mathbb{R}^{n}\right)$ into $w \in A_{\infty}\left(\mathbb{R}^{n}\right)$ with the sharp range $q(w) \in\left[1, \frac{n}{n-1}\right)$, where $q(w)$ denotes the critical index of the weight $w$ as in (1.6). Moreover, if we let $\varphi(x, t):=w(x) t^{p}$, with $w \in \mathrm{A}_{\infty}\left(\mathbb{R}^{n}\right)$ and $p \in\left(\frac{q(w)(n-1)}{n}, 1\right]$, for all $(x, t) \in \mathbb{R}^{n} \times[0, \infty)$, then $\varphi$ also satisfies the assumptions of Theorem 1.5 and Theorem 1.5 with this $\varphi$ extends the results obtained by Wheeden in [41] from the case $p=1$ into the case $p<1$.

(iii) In the sense of (i) and (ii) of this remark, the range of $\frac{i(\varphi)}{q(\varphi)} \in\left(\frac{n-1}{n}, \infty\right)$ in Theorem 1.5 is the best possible for the first order Riesz transform characterization of $H_{\varphi}\left(\mathbb{R}^{n}\right)$.

As in the case of $H^{p}\left(\mathbb{R}^{n}\right)$, the proof of Theorem 1.5 depends on the delicate characterizations of $H_{\varphi}\left(\mathbb{R}^{n}\right)$ via some harmonic functions and vectors defined on the upper half space $\mathbb{R}_{+}^{n+1}$. To this end, we first introduce the Musielak-Orlicz-Hardy spaces $H_{\varphi}\left(\mathbb{R}_{+}^{n+1}\right)$ of harmonic functions (see Definition 1.2 below) and $\mathcal{H}_{\varphi}\left(\mathbb{R}_{+}^{n+1}\right)$ of harmonic vectors (see Definition 2.10 below). However, unlike in the unweighted case, we cannot obtain the isomorphisms among $H_{\varphi}\left(\mathbb{R}^{n}\right), H_{\varphi}\left(\mathbb{R}_{+}^{n+1}\right)$ and $\mathcal{H}_{\varphi}\left(\mathbb{R}_{+}^{n+1}\right)$ without additional assumptions on $\varphi$. To remedy this, we introduce two subspace spaces, $H_{\varphi, 2}\left(\mathbb{R}_{+}^{n+1}\right)$ and $\mathcal{H}_{\varphi, 2}\left(\mathbb{R}_{+}^{n+1}\right)$, respectively, of $H_{\varphi}\left(\mathbb{R}_{+}^{n+1}\right)$ and $\mathcal{H}_{\varphi}\left(\mathbb{R}_{+}^{n+1}\right)$ (see Definitions 2.7 and 2.10 for their definitions). Then we establish the isomorphisms among $H_{\varphi}\left(\mathbb{R}^{n}\right), H_{\varphi, 2}\left(\mathbb{R}_{+}^{n+1}\right)$ and $\mathcal{H}_{\varphi, 2}\left(\mathbb{R}_{+}^{n+1}\right.$ ) (see Theorem 2.18 below).

With these results as preparation, let us sketch the proof of Theorem 1.5. To prove the inclusion $H_{\varphi}$, Riesz $\left(\mathbb{R}^{n}\right) \subset H_{\varphi}\left(\mathbb{R}^{n}\right)$, for any $f \in \mathbb{H}_{\varphi}$, Riesz $\left(\mathbb{R}^{n}\right)$, we construct a generalized Cauchy-Riemann system via the (conjugate) Poisson integrals of $f$ and $R_{j}(f)$, which is 
proved to be in $\mathcal{H}_{\varphi, 2}\left(\mathbb{R}_{+}^{n+1}\right)$. This, together with the isomorphism between $H_{\varphi}\left(\mathbb{R}^{n}\right)$ and $\mathcal{H}_{\varphi, 2}\left(\mathbb{R}_{+}^{n+1}\right)$, shows $H_{\varphi, \operatorname{Riesz}}\left(\mathbb{R}^{n}\right) \subset H_{\varphi}\left(\mathbb{R}^{n}\right)$.

To prove the inverse inclusion, we only need the radial maximal function characterization of $H_{\varphi}\left(\mathbb{R}^{n}\right)$ and the boundedness of Riesz transforms on $H_{\varphi}\left(\mathbb{R}^{n}\right)$ (see Proposition 2.2 and Corollary 2.22 below). Here, to prove Corollary 2.22, we establish an interpolation of operators on weighted Hardy spaces (see Proposition 2.21 below), which might be useful in establishing the boundedness of other important operators on $H_{\varphi}\left(\mathbb{R}^{n}\right.$ ) (see Corollary 2.23 below). We should mention that it is also possible to show Corollary 2.22 directly via the atomic and the molecular characterizations of $H_{\varphi}\left(\mathbb{R}^{n}\right)$, respectively, in [23, Theorem 1.1] and [17, Theorem 4.13]. However, the approach used in this article brings us more useful byproducts which have wide applications (see Proposition 2.21 and its applications below).

We now turn to the study of higher order Riesz transform characterizations of $H_{\varphi}\left(\mathbb{R}^{n}\right)$. Recall that there are several different approaches to introduce the higher order Riesz transforms (see, for example, [20]). In the present article, we focus on two kinds of higher order Riesz transforms: i) the higher order Riesz transforms which are compositions of first order Riesz transforms; ii) the higher order Riesz transforms defined via homogenous harmonic polynomials.

We start with the first one. Here, to simplify the notation, we restrict ourselves to $H_{\varphi}\left(\mathbb{R}^{n}\right) \cap L^{2}\left(\mathbb{R}^{n}\right)$.

Theorem 1.7. Let $m \in \mathbb{N} \cap[2, \infty)$ and $\varphi$ satisfy Assumption $(\varphi)$ with $\frac{i(\varphi)}{q(\varphi)}>\frac{n-1}{n+m-1}$, where $i(\varphi)$ and $q(\varphi)$ are as in (1.5) and (1.6), respectively. Assume further that $f \in$ $L^{2}\left(\mathbb{R}^{n}\right)$. Then $f \in H_{\varphi}\left(\mathbb{R}^{n}\right)$ if and only if there exists a positive constant $A$ such that, for all $k \in\{1, \ldots, m\}$ and $\left\{j_{1}, \ldots, j_{k}\right\} \subset\{1, \ldots, n\}, f, R_{j_{1}} \cdots R_{j_{k}}(f) \in L^{\varphi}\left(\mathbb{R}^{n}\right)$ and

$$
\|f\|_{L^{\varphi}\left(\mathbb{R}^{n}\right)}+\sum_{k=1}^{m} \sum_{j_{1}, \ldots, j_{k}=1}^{n}\left\|R_{j_{1}} \cdots R_{j_{k}}(f)\right\|_{L^{\varphi}\left(\mathbb{R}^{n}\right)} \leq A .
$$

Moreover, there exists a positive constant $C$, independent of $f$, such that

$$
\frac{1}{C}\|f\|_{H_{\varphi}\left(\mathbb{R}^{n}\right)} \leq A \leq C\|f\|_{H_{\varphi}\left(\mathbb{R}^{n}\right)} .
$$

Remark 1.8. (i) Let $m, k \in \mathbb{N}$ and $\left\{j_{1}, \ldots, j_{m}\right\} \subset\{0, \ldots, n\}$ satisfy that the number of the non-zero elements in $\left\{j_{1}, \ldots, j_{m}\right\}$ is $k$. Assume further that $R_{0}:=I$ is the identity operator. Then, we call $R_{j_{1}} \cdots R_{j_{m}}$ a $k$-order Riesz transform. Theorem 1.7 implies that, to obtain the Riesz transform characterization of $H_{\varphi}\left(\mathbb{R}^{n}\right)$ for all $\varphi$ satisfying $\frac{i(\varphi)}{q(\varphi)}>\frac{n-1}{n+m-1}$, we need all the $k$-order Riesz transforms for all $k \in\{0, \ldots, m\}$.

(ii) Compared with the first order Riesz transform characterization in Theorem 1.5. the higher order Riesz transform characterization in Theorem 1.7 does have some advantages. For example, we can relax the restrictions of $\varphi$ on both the type and the weight assumptions. To be more precise, by letting $m$ sufficiently large, one can obtain the Riesz transform characterization of $H_{\varphi}\left(\mathbb{R}^{n}\right)$ for any given $\varphi$ satisfying Assumption $(\varphi)$.

The scheme of the proof of Theorem 1.7 is similar to that of Theorem 1.5, The main difference is to replace the space $\mathcal{H}_{\varphi}\left(\mathbb{R}_{+}^{n+1}\right)$ by the Musielak-Orlicz-Hardy space $\mathcal{H}_{\varphi, m}\left(\mathbb{R}_{+}^{n+1}\right)$ 
of tensor-valued functions (see Definition 3.1 below), since, in this case, we have to make use of all Riesz transforms up to order $m$.

Now, we consider the second kind of higher Riesz transforms from Stein [31]. Let $f \in \mathcal{S}\left(\mathbb{R}^{n}\right), k \in \mathbb{N}$ and $\mathcal{P}_{k}$ be a homogenous harmonic polynomial of degree $k$. The Riesz transform of $f$ of degree $k$ associated with $\mathcal{P}_{k}$ is defined by setting, for all $x \in \mathbb{R}^{n}$,

$$
\mathcal{R}^{\mathcal{P}_{k}}(f)(x):=\lim _{\epsilon \rightarrow 0^{+}} \int_{|y| \geq \epsilon} \frac{\mathcal{P}_{k}(y)}{|y|^{n+k}} f(x-y) d y .
$$

For more details on homogenous harmonic polynomials, we refer the reader to [31, Section 3 of Chapter 3].

Furthermore, Kurokawa [20] obtained the following relationships between two kinds of higher Riesz transforms as above.

Proposition $1.9([20])$. Let $m, k \in \mathbb{N}$ and $\left\{j_{1}, \ldots, j_{m}\right\} \subset\{0, \ldots, n\}$ satisfy that the number of the non-zero elements in $\left\{j_{1}, \ldots, j_{m}\right\}$ is $k$. Let $f \in L^{2}\left(\mathbb{R}^{n}\right)$. Then, for each $k$-order Riesz transform $R_{j_{1}} \cdots R_{j_{m}}$ as in Remark 1.8, there exist $\ell \in \mathbb{N}$ and a positive constant $C$ such that

$$
R_{j_{1}} \cdots R_{j_{m}}(f)=C f+(-1)^{k} \sum_{j=0}^{\ell} \mathcal{R}^{\mathcal{P}_{j}}(f),
$$

where $\mathcal{P}_{j}$ ranges over all the homogenous harmonic polynomials of degree $k-2 j$ and $\mathcal{R}^{\mathcal{P}_{j}}$ is the higher order Riesz transform of degree $k-2 j$ associated with $\mathcal{P}_{j}$ defined as in (1.11).

Combining Proposition 1.9 and Theorem 1.7, we conclude the following corollary, which establishes the Riesz transform characterization of $H_{\varphi}\left(\mathbb{R}^{n}\right)$ in terms of higher Riesz transforms defined via homogenous harmonic polynomials.

Corollary 1.10. Let $m \in \mathbb{N} \cap[2, \infty), k \in\{0, \ldots, m\}$ and $\varphi$ satisfy Assumption $(\varphi)$ with $\frac{i(\varphi)}{q(\varphi)}>\frac{n-1}{n+m-1}$, where $i(\varphi)$ and $q(\varphi)$ are as in (1.5) and (1.6), respectively. Suppose that $f \in L^{2}\left(\mathbb{R}^{n}\right)$. Then $f \in H_{\varphi}\left(\mathbb{R}^{n}\right)$ if and only if there exists a positive constant $A$ such that, for all homogenous harmonic polynomials $\mathcal{P}_{j}$ of degree $k, f, \mathcal{R}^{\mathcal{P}_{j}}(f) \in L^{\varphi}\left(\mathbb{R}^{n}\right)$ and

$$
\|f\|_{L^{\varphi}\left(\mathbb{R}^{n}\right)}+\sum_{j}\left\|\mathcal{R}^{\mathcal{P}_{j}}(f)\right\|_{L^{\varphi}\left(\mathbb{R}^{n}\right)} \leq A
$$

Moreover, there exists a positive constant $C$, independent of $f$, such that

$$
\frac{1}{C}\|f\|_{H_{\varphi}\left(\mathbb{R}^{n}\right)} \leq A \leq C\|f\|_{H_{\varphi}\left(\mathbb{R}^{n}\right)},
$$

where $\mathcal{P}_{j}$ ranges over all the homogenous harmonic polynomials of degree $k$ with $k \in$ $\{0, \ldots, m\}$.

Observe that, in Corollary 1.10, we use less Riesz transforms than Theorem 1.7 to characterize $H_{\varphi}\left(\mathbb{R}^{n}\right)$, since not every polynomial of order $k$ is homogeneous harmonic. 
Moreover, there arises a natural question for Riesz transform characterizations of $H_{\varphi}\left(\mathbb{R}^{n}\right)$ : in these characterizations, can we use Riesz transforms as less as possible? This question can not be solved directly by the methods used to prove Theorems [1.5] and 1.7, because the heart of these methods relies on the subharmonic property of the absolute value of a harmonic vector (resp. tensor-valued function) satisfying the generalized Cauchy-Riemann equation. Moreover, to construct such a harmonic vector (resp. tensor-valued function), we always need all the Riesz transforms up to a fixed order.

Corollary 1.10 provides a method to solve the above problem via replacing all Riesz transforms up to order $m$ by Riesz transforms defined via homogenous harmonic polynomials. Another method is from Uchiyama [38, 39], which avoids the use of the subharmonic property by using the Fourier multiplier and has a close relationship with the constructive proof of the Fefferman-Stein decomposition of $\mathrm{BMO}\left(\mathbb{R}^{n}\right)$.

The following theorem establishes the odd order Riesz transform characterization of $H_{\varphi}\left(\mathbb{R}^{n}\right)$ based on the method of Uchiyama.

Theorem 1.11. Let $k \in \mathbb{N}$ be odd, $\varphi$ satisfy Assumption $(\varphi)$ and $\frac{i(\varphi)}{q(\varphi)}>\max \left\{p_{0}, \frac{1}{2}\right\}$, where $i(\varphi), q(\varphi)$ and $p_{0}$ are, respectively, as in (1.5), (1.6) and Proposition 3.7 below. Let $f \in L^{2}\left(\mathbb{R}^{n}\right)$. Then $f \in H_{\varphi}\left(\mathbb{R}^{n}\right)$ if and only if, for all $\left\{j_{1}, \ldots, j_{k}\right\} \subset\{1, \ldots, n\}, f$ and $R_{j_{1}} \cdots R_{j_{k}}(f) \in L^{\varphi}\left(\mathbb{R}^{n}\right)$. Moreover, there exists a positive constant $C$, independent of $f$, such that

$$
\begin{aligned}
\frac{1}{C}\|f\|_{H_{\varphi}\left(\mathbb{R}^{n}\right)} & \leq\|f\|_{L^{\varphi}\left(\mathbb{R}^{n}\right)}+\sum_{j_{1}, \ldots, j_{k}=1}^{n}\left\|R_{j_{1}} \cdots R_{j_{k}}(f)\right\|_{L^{\varphi}\left(\mathbb{R}^{n}\right)} \\
& \leq C\|f\|_{H_{\varphi}\left(\mathbb{R}^{n}\right)} \cdot
\end{aligned}
$$

Remark 1.12. (i) We point out that, in [8], Fefferman conjectured that "nice" conjugate systems, such as the second order Riesz transforms, would also give a characterization of $H^{1}\left(\mathbb{R}^{2}\right)$. However, Gandulfo, García-Cuerva and Taibleson [10] have constructed a counterexample to show that even order Riesz transforms fail to characterize $H^{1}\left(\mathbb{R}^{2}\right)$. This justifies the characterization of $H_{\varphi}\left(\mathbb{R}^{n}\right)$ via odd order Riesz transforms.

(ii) Compared with Corollary 1.10, in Theorem 1.11, we use much less Riesz transforms. However, a shortcoming is that we can only deal with the case $\frac{i(\varphi)}{q(\varphi)}>\max \left\{p_{0}, \frac{1}{2}\right\}$, where $p_{0} \in(0,1)$ is described in Proposition 3.7 below.

(iii) We point out that Theorem 1.7, Corollary 1.10 and Theorem 1.11 have variants as in Theorem 1.5, the details being omitted.

The organization of this article is as follows.

In Section 2, we give out the proof of Theorem 1.5. To this end, we establish some necessary and auxiliary results. More precisely, in Subsection 2.1, we establish the radial maximal function and the Poisson integral characterizations of $H_{\varphi}\left(\mathbb{R}^{n}\right)$ (see Propositions 2.2 and 2.4 below).

In Subsection 2.2, we introduce a Musielak-Orlicz-Hardy space $H_{\varphi}\left(\mathbb{R}_{+}^{n+1}\right)$ of harmonic functions (see Definition 1.2 below) and show that the subspace $H_{\varphi, 2}\left(\mathbb{R}_{+}^{n+1}\right)$ of $H_{\varphi}\left(\mathbb{R}_{+}^{n+1}\right.$ ) is isomorphic to $H_{\varphi}\left(\mathbb{R}^{n}\right)$ (see Proposition 2.9 below).

In Subsection 2.3, we introduce a Musielak-Orlicz-Hardy space $\mathcal{H}_{\varphi}\left(\mathbb{R}_{+}^{n+1}\right)$ of harmonic vectors which satisfy the generalized Cauchy-Riemann equation (2.16) (see Definition 2.10 
below), then we show that the elements in $\mathcal{H}_{\varphi}\left(\mathbb{R}_{+}^{n+1}\right)$ have harmonic majorant and boundary value on $\mathbb{R}^{n}$ (see Lemmas 2.12 and 2.13 below). Moreover, by establishing relations among $\mathcal{H}_{\varphi}\left(\mathbb{R}_{+}^{n+1}\right), H_{\varphi}\left(\mathbb{R}_{+}^{n+1}\right)$ and $H_{\varphi}\left(\mathbb{R}^{n}\right)$ (see Propositions 2.15 and 2.17 below), we obtain the isomorphisms among the spaces $H_{\varphi}\left(\mathbb{R}^{n}\right), H_{\varphi, 2}\left(\mathbb{R}_{+}^{n+1}\right)$ and $\mathcal{H}_{\varphi, 2}\left(\mathbb{R}_{+}^{n+1}\right.$ ) (see Theorem 2.18 below).

In Subsection 2.4, we prove Theorem 1.5 via Theorem 2.18, Furthermore, we also need the boundedness of Riesz transforms on $H_{\varphi}\left(\mathbb{R}^{n}\right)$ (see Corollary 2.22 below), which is proved by establishing an interpolation of operators on weighted Hardy spaces (see Proposition 2.21 below). We point out that this interpolation result may be of independent interest, since, by which, we can obtain the boundedness of many important operators from harmonic analysis and partial differential equations on $H_{\varphi}\left(\mathbb{R}^{n}\right)$; see Corollary 2.23 for the case of Calderón-Zygmund operators.

In Section 3, we first introduce a Musielak-Orlicz-Hardy space $\mathcal{H}_{\varphi, m}\left(\mathbb{R}_{+}^{n+1}\right)$ of tensorvalued functions (see Definition 3.1 below), which plays the same role as $\mathcal{H}_{\varphi}\left(\mathbb{R}_{+}^{n+1}\right)$ in the first order Riesz transform characterizations (see Remark 1.8(ii) below). Then we prove Theorem 1.7 by a way similar to that used in the proof of Theorem 1.5. Finally, by using an estimate of Uchiyama [38, we prove Theorem 1.11.

We end this section by making some conventions on notation. Throughout the whole article, we always set $\mathbb{N}:=\{1,2, \ldots\}$ and $\mathbb{Z}_{+}:=\mathbb{N} \cup\{0\}$. The differential operator $\frac{\partial^{|\alpha|}}{\partial x_{1}^{\alpha_{1}} \ldots \partial x_{n}^{\alpha_{n}}}$ is denoted simply by $\partial^{\alpha}$, where $\alpha:=\left(\alpha_{1}, \ldots, \alpha_{n}\right) \in \mathbb{Z}_{+}^{n}$ and $|\alpha|:=\alpha_{1}+\cdots+\alpha_{n}$. Let $C_{c}^{\infty}\left(\mathbb{R}^{n}\right)$ be the set of smooth functions with compact support. We use $C$ to denote a positive constant that is independent of the main parameters involved but whose value may differ from line to line. We use $C_{(\alpha, \beta, \ldots)}$ to denote a positive constant depending on the parameters $\alpha, \beta \ldots$ If $f \leq C g$, we then write $f \lesssim g$ and, if $f \lesssim g \lesssim f$, we then write $f \sim g$. For all $x \in \mathbb{R}^{n}$ and $r \in(0, \infty)$, let $B(x, r):=\left\{y \in \mathbb{R}^{n}:|x-y|<r\right\}$. Also, for any set $E \subset \mathbb{R}^{n}$, we use $E^{\complement}$ to denote $\mathbb{R}^{n} \backslash E$ and $\chi_{E}$ its characteristic function, respectively. For any $s \in \mathbb{R}$, we let $\lfloor s\rfloor$ to denote the maximal integer not more than $s$. Finally, for $q \in[1, \infty], q^{\prime}:=\frac{q}{q-1}$ denotes the conjugate exponent of $q$.

\section{First order Riesz transform characterizations}

In this section, we give a complete proof of Theorem 1.5. In order to achieve this goal, we need to introduce Musielak-Orlicz-Hardy type spaces $H_{\varphi}\left(\mathbb{R}_{+}^{n+1}\right)$ of harmonic functions and $\mathcal{H}_{\varphi}\left(\mathbb{R}_{+}^{n+1}\right)$ of harmonic vectors on the upper half space $\mathbb{R}_{+}^{n+1}$, and establish their relations with $H_{\varphi}\left(\mathbb{R}^{n}\right)$. The first three subsections of this section are devoted to the study of these relations. After this, we prove Theorem [1.5] in Subsection 2.4.

\subsection{Radial maximal function and Poisson integral characterizations of $H_{\varphi}\left(\mathbb{R}^{n}\right)$}

Let $p \in[1, \infty)$ and $w \in A_{p}\left(\mathbb{R}^{n}\right)$. It is well known that there exist positive constants $\delta \in(0,1)$ and $C$ such that, for all balls $B_{1}, B_{2} \subset \mathbb{R}^{n}$ with $B_{1} \subset B_{2}$,

$$
\frac{w\left(B_{2}\right)}{w\left(B_{1}\right)} \leq C\left(\frac{\left|B_{2}\right|}{\left|B_{1}\right|}\right)^{p}
$$


and

$$
\frac{w\left(B_{1}\right)}{w\left(B_{2}\right)} \leq C\left(\frac{\left|B_{1}\right|}{\left|B_{2}\right|}\right)^{\delta}
$$

(see, for example, 12 for more details on the above two inequalities and other properties of Muckenhoupt weights).

Now, let $\phi \in \mathcal{S}\left(\mathbb{R}^{n}\right)$ satisfy

$$
\int_{\mathbb{R}^{n}} \phi(x) d x=1
$$

For any distribution $f \in \mathcal{S}^{\prime}\left(\mathbb{R}^{n}\right)$, its radial and non-tangential maximal functions $\mathcal{M}_{\phi}(f)$ and $\mathcal{M}_{\phi}^{*}(f)$ are, respectively, defined by setting, for all $x \in \mathbb{R}^{n}$,

$$
\mathcal{M}_{\phi}(f)(x):=\sup _{t \in(0, \infty)}\left|\left(f * \phi_{t}\right)(x)\right|
$$

and

$$
\mathcal{M}_{\phi}^{*}(f)(x):=\sup _{|y-x|<t, t \in(0, \infty)}\left|\left(f * \phi_{t}\right)(y)\right| .
$$

Liang, Huang and Yang [24, Theorem 3.7] established the following non-tangential maximal function characterization of $H_{\varphi}\left(\mathbb{R}^{n}\right)$.

Proposition 2.1 ([24]). Let $\varphi$ and $\phi \in \mathcal{S}\left(\mathbb{R}^{n}\right)$ satisfy, respectively, Assumption $(\varphi)$ and (2.3). Then $f \in H_{\varphi}\left(\mathbb{R}^{n}\right)$ if and only if $f \in \mathcal{S}^{\prime}\left(\mathbb{R}^{n}\right)$ and $\mathcal{M}_{\phi}^{*}(f) \in L^{\varphi}\left(\mathbb{R}^{n}\right)$. Moreover, there exists a positive constant $C$ such that, for all $f \in H_{\varphi}\left(\mathbb{R}^{n}\right)$,

$$
\frac{1}{C}\|f\|_{H_{\varphi}\left(\mathbb{R}^{n}\right)} \leq\left\|\mathcal{M}_{\phi}^{*}(f)\right\|_{L^{\varphi}\left(\mathbb{R}^{n}\right)} \leq C\|f\|_{H_{\varphi}\left(\mathbb{R}^{n}\right)} .
$$

The following result provides the radial maximal function characterization of $H_{\varphi}\left(\mathbb{R}^{n}\right)$.

Proposition 2.2. Let $\varphi$ and $\phi \in \mathcal{S}\left(\mathbb{R}^{n}\right)$ satisfy, respectively, Assumption $(\varphi)$ and (2.3). Then $f \in H_{\varphi}\left(\mathbb{R}^{n}\right)$ if and only if $f \in \mathcal{S}^{\prime}\left(\mathbb{R}^{n}\right)$ and $\mathcal{M}_{\phi}(f) \in L^{\varphi}\left(\mathbb{R}^{n}\right)$. Moreover, there exists a positive constant $C$ such that, for all $f \in H_{\varphi}\left(\mathbb{R}^{n}\right)$,

$$
\frac{1}{C}\|f\|_{H_{\varphi}\left(\mathbb{R}^{n}\right)} \leq\left\|\mathcal{M}_{\phi}(f)\right\|_{L^{\varphi}\left(\mathbb{R}^{n}\right)} \leq C\|f\|_{H_{\varphi}\left(\mathbb{R}^{n}\right)} .
$$

To prove Proposition 2.2, we need the following boundedness of the Hardy-Littlewood maximal function on $L^{\varphi}\left(\mathbb{R}^{n}\right)$ from [24, Corollary 2.8]. Recall that, for all $x \in \mathbb{R}^{n}$, the Hardy-Littlewood maximal function $\mathcal{M}(f)$ of a locally integrable function $f$ on $\mathbb{R}^{n}$ is defined by setting,

$$
\mathcal{M}(f)(x):=\sup _{B \ni x} \frac{1}{|B|} \int_{B}|f(y)| d y
$$

where the supremum is taken over all balls $B$ in $\mathbb{R}^{n}$ containing $x$. 
Lemma $2.3([24])$. Let $\varphi$ satisfy Assumption $(\varphi)$ with the lower type exponent $p \in(1, \infty)$ and $q(\varphi)<i(\varphi)$, where $q(\varphi)$ and $i(\varphi)$ are as in (1.6) and (1.5), respectively. Then $\mathcal{M}$ is bounded on $L^{\varphi}\left(\mathbb{R}^{n}\right)$. Moreover, there exists a positive constant $C$ such that, for all $f \in L^{\varphi}\left(\mathbb{R}^{n}\right)$,

$$
\int_{\mathbb{R}^{n}} \varphi(x, \mathcal{M}(f)(x)) d x \leq C \int_{\mathbb{R}^{n}} \varphi(x,|f(x)|) d x .
$$

We now turn to the proof of Proposition 2.2 .

Proof of Proposition 2.2. The direction that $f \in H_{\varphi}\left(\mathbb{R}^{n}\right)$ implies $\mathcal{M}_{\phi}(f) \in L^{\varphi}\left(\mathbb{R}^{n}\right)$ is an easy consequence of Proposition 2.1 and the fact that, for all $x \in \mathbb{R}^{n}, \mathcal{M}_{\phi}(f)(x) \leq$ $\mathcal{M}_{\phi}^{*}(f)(x)$, the details being omitted.

Now, let $f \in \mathcal{S}^{\prime}\left(\mathbb{R}^{n}\right)$ satisfy $\mathcal{M}_{\phi}(f) \in L^{\varphi}\left(\mathbb{R}^{n}\right)$. Based on Proposition 2.1, we prove another direction of Proposition 2.2 by showing that

$$
\left\|\mathcal{M}_{\phi}^{*}(f)\right\|_{L^{\varphi}\left(\mathbb{R}^{n}\right)} \lesssim\left\|\mathcal{M}_{\phi}(f)\right\|_{L^{\varphi}\left(\mathbb{R}^{n}\right)} .
$$

Indeed, for any $\epsilon \in(0,1), N \in \mathbb{N}$ sufficiently large and $x \in \mathbb{R}^{n}$, let

$$
\mathcal{M}_{\phi, \epsilon, N}^{*}(f)(x):=\sup _{|x-y|<t<\frac{1}{\epsilon}}\left|\left(f * \phi_{t}\right)(y)\right|\left(\frac{t}{t+\epsilon}\right)^{N}(1+\epsilon|y|)^{-N} .
$$

It is easy to see that, for all $x \in \mathbb{R}^{n}, \lim _{\epsilon \rightarrow 0^{+}, N \rightarrow \infty} \mathcal{M}_{\phi, \epsilon, N}^{*}(f)(x)=\mathcal{M}_{\phi}^{*}(f)(x)$.

We first claim that, for all $\lambda \in(0, \infty)$, there exists a positive constant $C_{(N, n, \varphi, \phi)}$, depending only on $N, n, \varphi$ and $\phi$, such that

$$
\int_{\mathbb{R}^{n}} \varphi\left(x, \frac{\mathcal{M}_{\phi, \epsilon, N}^{*}(f)(x)}{\lambda}\right) d x \leq C_{(N, n, \varphi, \phi)} \int_{\mathbb{R}^{n}} \varphi\left(x, \frac{\mathcal{M}_{\phi}(f)(x)}{\lambda}\right) d x .
$$

To prove this claim, for all $x \in \mathbb{R}^{n}$, let

$$
\widetilde{\mathcal{M}}_{\phi, \epsilon, N}^{*}(f)(x):=\sup _{|x-y|<t<\frac{1}{\epsilon}} t\left|\nabla_{y}\left(f * \phi_{t}\right)(y)\right|\left(\frac{t}{t+\epsilon}\right)^{N}(1+\epsilon|y|)^{-N} .
$$

From the proof of [14, (6.4.22)], we deduce that, for any $p \in(0, \infty), \epsilon \in(0,1)$ and $N \in \mathbb{N}$, there exists a positive constant $C_{(N, n, \varphi, \phi)}$ such that, for all $x \in \mathbb{R}^{n}$,

$$
\widetilde{\mathcal{M}}_{\phi, \epsilon, N}^{*}(f)(x) \leq C_{(N, n, \varphi, \phi)}\left\{\mathcal{M}\left(\left[\mathcal{M}_{\phi, \epsilon, N}^{*}(f)\right]^{p}\right)(x)\right\}^{1 / p}
$$

where $\mathcal{M}$ denotes the Hardy-Littlewood maximal function as in (2.6).

Now, let

$$
E_{\epsilon, N}:=\left\{x \in \mathbb{R}^{n}: \widetilde{\mathcal{M}}_{\phi, \epsilon, N}^{*}(f)(x) \leq C_{0} \mathcal{M}_{\phi, \epsilon, N}^{*}(f)(x)\right\},
$$

where $C_{0}$ is a sufficiently large constant whose size will be determined later. For all $(x, t) \in \mathbb{R}_{+}^{n+1}$, let $\varphi_{p}(x, t):=\varphi\left(x, t^{1 / p}\right)$. By the definition of $i(\varphi)$, we know that there 
exists $p_{0} \in(0, i(\varphi))$ such that, for any $x \in \mathbb{R}^{n}, \varphi(x, \cdot)$ is of lower type $p_{0}$. It is easy to see that $i\left(\varphi_{p}\right)=\frac{i(\varphi)}{p}$ and, for any $x \in \mathbb{R}^{n}, \varphi_{p}(x, \cdot)$ is of lower type $\frac{p_{0}}{p}$. Thus, by taking $p$ sufficiently small, we obtain $q\left(\varphi_{p}\right)<i\left(\varphi_{p}\right)$, which, together with (2.9), Lemma 2.3 and the lower type $p_{0}$ property of $\varphi(x, \cdot)$, implies that there exists a positive constant $C_{(\varphi)}$ satisfying that, for any $\lambda \in(0, \infty)$,

$$
\begin{aligned}
& \int_{\left(E_{\epsilon, N}\right)^{\complement}} \varphi\left(x, \frac{\mathcal{M}_{\phi, \epsilon, N}^{*}(f)(x)}{\lambda}\right) d x \\
& \leq C_{(\varphi)}\left(\frac{1}{C_{0}}\right)^{p_{0}} \int_{\left(E_{\epsilon, N}\right)^{\complement}} \varphi\left(x, \frac{\widetilde{\mathcal{M}}_{\phi, \epsilon, N}^{*}(f)(x)}{\lambda}\right) d x \\
& \leq C_{(N, n, \varphi, \phi)}\left(\frac{1}{C_{0}}\right)^{p_{0}} \int_{\left(E_{\epsilon, N}\right)^{\complement}} \varphi_{p}\left(x, \frac{\mathcal{M}\left(\left[\mathcal{M}_{\phi, \epsilon, N}^{*}(f)\right]^{p}\right)(x)}{\lambda^{p}}\right) d x \\
& \leq C_{(N, n, \varphi, \phi)}\left(\frac{1}{C_{0}}\right)^{p_{0}} \int_{\mathbb{R}^{n}} \varphi\left(x, \frac{\mathcal{M}_{\phi, \epsilon, N}^{*}(f)(x)}{\lambda}\right) d x .
\end{aligned}
$$

By taking $C_{0}$ in (2.10) sufficiently large so that $C_{(N, n, \varphi, \phi)}\left(\frac{1}{C_{0}}\right)^{p_{0}}<\frac{1}{2}$, we see that

$$
\int_{\mathbb{R}^{n}} \varphi\left(x, \frac{\mathcal{M}_{\phi, \epsilon, N}^{*}(f)(x)}{\lambda}\right) d x \leq 2 \int_{E_{\epsilon, N}} \varphi\left(x, \frac{\mathcal{M}_{\phi, \epsilon, N}^{*}(f)(x)}{\lambda}\right) d x .
$$

Moreover, from [14, (6.4.27)], it follows that, for all $r<i(\varphi)$ and $x \in E_{\epsilon, N}$,

$$
\mathcal{M}_{\phi, \epsilon, N}^{*}(f)(x) \leq C_{(N, n, \varphi, \phi)}\left\{\mathcal{M}\left(\left[\mathcal{M}_{\phi}(f)\right]^{r}\right)(x)\right\}^{1 / r},
$$

which, together with (2.11) and an argument similar to that used in the estimate (2.10), implies that (2.8) holds true.

Now, we finish the proof of Proposition 2.2 by using the above claim. Observe that, for $x \in \mathbb{R}^{n}$,

$$
\mathcal{M}_{\phi, \epsilon, N}^{*}(f)(x) \geq \frac{2^{-N}}{(1+\epsilon|x|)^{N}} \sup _{|x-y|<t<\frac{1}{\epsilon}}\left|\left(f * \phi_{t}\right)(y)\right|\left(\frac{t}{t+\epsilon}\right)^{N}=: F_{\epsilon, N}(x) .
$$

It is easy to see, for each $N$ and $x, F_{\epsilon, N}(x)$ is increasing to $2^{-N} \mathcal{M}_{\phi}^{*}(f)(x)$ as $\epsilon \rightarrow 0^{+}$, which, combined with (2.8) and Lebesgue's monotone convergence theorem, implies that

$$
\int_{\mathbb{R}^{n}} \varphi\left(x, \frac{\mathcal{M}_{\phi}^{*}(f)(x)}{\lambda}\right) d x \leq C_{(N, n, \varphi, \phi)} \int_{\mathbb{R}^{n}} \varphi\left(x, \frac{\mathcal{M}_{\phi}(f)(x)}{\lambda}\right) d x .
$$

In particular, $\mathcal{M}_{\phi}(f) \in L^{\varphi}\left(\mathbb{R}^{n}\right)$ implies that $\mathcal{M}_{\phi}^{*}(f) \in L^{\varphi}\left(\mathbb{R}^{n}\right)$. This, together with a repetition of the above argument used in the proof of the estimate (2.8) with $\epsilon:=0$ and $N:=\infty$ in $\mathcal{M}_{\phi, \epsilon, N}^{*}(f)$ and $\widetilde{\mathcal{M}}_{\phi, \epsilon, N}^{*}(f)$, implies that

$$
\int_{\mathbb{R}^{n}} \varphi\left(x, \frac{\mathcal{M}_{\phi}^{*}(f)(x)}{\lambda}\right) d x \leq C_{(n, \varphi, \phi)} \int_{\mathbb{R}^{n}} \varphi\left(x, \frac{\mathcal{M}_{\phi}(f)(x)}{\lambda}\right) d x .
$$

This finishes the proof of Proposition 2.2. 
We also need the following Poisson integral characterization of $H_{\varphi}\left(\mathbb{R}^{n}\right)$. Recall that a distribution $f \in \mathcal{S}^{\prime}\left(\mathbb{R}^{n}\right)$ is called a bounded distribution, if, for any $\phi \in \mathcal{S}\left(\mathbb{R}^{n}\right), f * \phi \in$ $L^{\infty}\left(\mathbb{R}^{n}\right)$. For all $(x, t) \in \mathbb{R}_{+}^{n+1}$, let

$$
P_{t}(x):=C_{(n)} \frac{t}{\left(t^{2}+|x|^{2}\right)^{(n+1) / 2}}
$$

be the Poisson kernel, where $C_{(n)}$ is the same as in (1.1). It is well known that, if $f$ is a bounded distribution, then $f * P_{t}$ is a well-defined, bounded and smooth function. Moreover, $f * P_{t}$ is harmonic on $\mathbb{R}_{+}^{n+1}$ (see [32, p. 90]).

Recall that, in [32, p. 91, Theorem 1], Stein established the Poisson integral characterization of the classical Hardy space $H^{p}\left(\mathbb{R}^{n}\right)$ by using some pointwise estimates. These estimates can directly be used in our setting to obtain the following proposition, the details being omitted.

Proposition 2.4. Let $\varphi$ satisfy Assumption $(\varphi)$ and $f \in \mathcal{S}^{\prime}\left(\mathbb{R}^{n}\right)$ be a bounded distribution. Then $f \in H_{\varphi}\left(\mathbb{R}^{n}\right)$ if and only if $f_{P}^{*} \in L^{\varphi}\left(\mathbb{R}^{n}\right)$, where, for all $x \in \mathbb{R}^{n}$,

$$
f_{P}^{*}(x):=\sup _{|y-x|<t, t \in(0, \infty)}\left|\left(f * P_{t}\right)(y)\right| .
$$

Moreover, there exists a positive constant $C$ such that, for all $f \in H_{\varphi}\left(\mathbb{R}^{n}\right)$,

$$
\frac{1}{C}\|f\|_{H_{\varphi}\left(\mathbb{R}^{n}\right)} \leq\left\|f_{P}^{*}\right\|_{L^{\varphi}\left(\mathbb{R}^{n}\right)} \leq C\|f\|_{H_{\varphi}\left(\mathbb{R}^{n}\right)} .
$$

Remark 2.5. We point out that the statement of Proposition 2.4 is a little bit different from that of $\left[32\right.$, p. 91 , Theorem 1] in that here we assume, a priori, that $f \in \mathcal{S}^{\prime}\left(\mathbb{R}^{n}\right)$ is a bounded distribution. This is because that, for an arbitrary $f \in H_{\varphi}\left(\mathbb{R}^{n}\right)$, we cannot show that $f$ is a bounded distribution without any additional assumptions on $f$ or $\varphi$. However, by the facts that the set $H_{\varphi}\left(\mathbb{R}^{n}\right) \cap L^{2}\left(\mathbb{R}^{n}\right)$ is dense in $H_{\varphi}\left(\mathbb{R}^{n}\right)$ and there exist $\psi_{1}, \psi_{2} \in \mathcal{S}\left(\mathbb{R}^{n}\right)$ and $h \in L^{1}\left(\mathbb{R}^{n}\right)$ such that, for all $t \in(0, \infty)$,

$$
P_{t}=\left(\psi_{1}\right)_{t} * h_{t}+\left(\psi_{2}\right)_{t}
$$

(see [32, p. 90]), we know that, for every $f \in H_{\varphi}\left(\mathbb{R}^{n}\right)$, we can define $f * P_{t}$ by setting, for all $x \in \mathbb{R}^{n}$ and $t \in(0, \infty),\left(f * P_{t}\right)(x):=\lim _{k \rightarrow \infty}\left(f_{k} * P_{t}\right)(x)$, where $\left\{f_{k}\right\}_{k \in \mathbb{N}} \subset$ $\left(H_{\varphi}\left(\mathbb{R}^{n}\right) \cap L^{2}\left(\mathbb{R}^{n}\right)\right)$ satisfies $\lim _{k \rightarrow \infty} f_{k}=f$ in $H_{\varphi}\left(\mathbb{R}^{n}\right)$ and hence in $\mathcal{S}^{\prime}\left(\mathbb{R}^{n}\right)$.

\subsection{Musielak-Orlicz-Hardy spaces $H_{\varphi}\left(\mathbb{R}_{+}^{n+1}\right)$ of harmonic functions}

In this subsection, we introduce the Musielak-Orlicz-Hardy space $H_{\varphi}\left(\mathbb{R}_{+}^{n+1}\right)$ of harmonic functions and establish its relation with $H_{\varphi}\left(\mathbb{R}^{n}\right)$.

To this end, let $u$ be a function on $\mathbb{R}_{+}^{n+1}$. Its non-tangential maximal function $u^{*}$ is defined by setting, for all $x \in \mathbb{R}^{n}$,

$$
u^{*}(x):=\sup _{|y-x|<t, t \in(0, \infty)}|u(y, t)| .
$$

Recall that a function $u$ on $\mathbb{R}_{+}^{n+1}$ is said to be harmonic if $\left(\Delta_{x}+\partial_{t}^{2}\right) u(x, t)=0$ for all $(x, t) \in \mathbb{R}_{+}^{n+1}$. 
Definition 2.6. Let $\varphi$ satisfy Assumption $(\varphi)$. The Musielak-Orlicz-Hardy space of harmonic functions, $H_{\varphi}\left(\mathbb{R}_{+}^{n+1}\right)$, is defined to be the space of all harmonic functions $u$ on $\mathbb{R}_{+}^{n+1}$ such that $u^{*} \in L^{\varphi}\left(\mathbb{R}^{n}\right)$. Moreover, for all $u \in H_{\varphi}\left(\mathbb{R}_{+}^{n+1}\right)$, its quasi-norm is defined by $\|u\|_{H_{\varphi}\left(\mathbb{R}_{+}^{n+1}\right)}:=\left\|u^{*}\right\|_{L^{\varphi}\left(\mathbb{R}^{n}\right)}$.

Recall also the following notion of the Hardy space $H^{p}\left(\mathbb{R}_{+}^{n+1}\right)$ of harmonic functions with $p \in(1, \infty)$ from [35] (see also [2]).

Definition 2.7 ([35]). Let $p \in(1, \infty)$. The Hardy space $H^{p}\left(\mathbb{R}_{+}^{n+1}\right)$ of harmonic functions is defined to be the space of all harmonic functions $u$ on $\mathbb{R}_{+}^{n+1}$ such that, for all $t \in(0, \infty)$, $u(\cdot, t) \in L^{p}\left(\mathbb{R}^{n}\right)$. Moreover, for all $u \in H^{p}\left(\mathbb{R}_{+}^{n+1}\right)$, its norm is defined by

$$
\|u\|_{H^{p}\left(\mathbb{R}_{+}^{n+1}\right)}:=\sup _{t \in(0, \infty)}\|u(\cdot, t)\|_{L^{p}\left(\mathbb{R}^{n}\right)} .
$$

For $\varphi$ as in Definition 2.6, let

$$
H_{\varphi, 2}\left(\mathbb{R}_{+}^{n+1}\right):=\overline{H_{\varphi}\left(\mathbb{R}_{+}^{n+1}\right) \cap H^{2}\left(\mathbb{R}_{+}^{n+1}\right)}\|\cdot\|_{H_{\varphi}\left(\mathbb{R}_{+}^{n+1}\right)}
$$

be the completion of the set $H_{\varphi}\left(\mathbb{R}_{+}^{n+1}\right) \cap H^{2}\left(\mathbb{R}_{+}^{n+1}\right)$ under the quasi-norm $\|\cdot\|_{H_{\varphi}\left(\mathbb{R}_{+}^{n+1}\right)}$.

Remark 2.8. For any $u \in H_{\varphi}\left(\mathbb{R}_{+}^{n+1}\right) \cap H^{2}\left(\mathbb{R}_{+}^{n+1}\right)$, from the Poisson integral characterization of $H^{2}\left(\mathbb{R}_{+}^{n+1}\right)$ (see, for example, [2, Theorem 7.17]), we deduce that $u$ satisfies the following semigroup formula that, for all $x \in \mathbb{R}^{n}$ and $s, t \in(0, \infty)$,

$$
u(x, s+t)=\left(u(\cdot, s) * P_{t}\right)(x),
$$

where $P_{t}$ denotes the Poisson kernel as in (2.12). This formula was first introduced by Bui in [5. Moreover, let $p \in(0,1], w \in A_{\infty}\left(\mathbb{R}^{n}\right)$ and $H_{w}^{p}\left(\mathbb{R}_{+}^{n+1}\right)$ be the weighted Hardy space of harmonic functions defined as in Definition 2.6 via the radial maximal functions. Let $\bar{H}_{w}^{p}\left(\mathbb{R}_{+}^{n+1}\right)$ be the closure in $H_{w}^{p}\left(\mathbb{R}_{+}^{n+1}\right)$ of the subspace of those functions in $H_{w}^{p}\left(\mathbb{R}_{+}^{n+1}\right)$ for which the semigroup formula (2.14) holds. Bui proposed the question that, under what condition, $\bar{H}_{w}^{p}\left(\mathbb{R}_{+}^{n+1}\right)$ is equivalent to $H_{w}^{p}\left(\mathbb{R}_{+}^{n+1}\right)$. It is known that if, for some $d \in(0, \infty)$, $w$ satisfies the following extra condition that, for all $x \in \mathbb{R}^{n}$ and $\rho \in(0,1)$,

$$
\int_{B(x, \rho)} w(y) d y \gtrsim \rho^{d}
$$

then $\bar{H}_{w}^{p}\left(\mathbb{R}_{+}^{n+1}\right)=H_{w}^{p}\left(\mathbb{R}_{+}^{n+1}\right)$ (see [5, Remark 3.3]). In particular, if $w \equiv 1$, the above two spaces coincide. We refer the reader to [5, Remark 3.3] for more details.

The following proposition shows that the spaces, $H_{\varphi, 2}\left(\mathbb{R}_{+}^{n+1}\right)$ and $H_{\varphi}\left(\mathbb{R}^{n}\right)$, are isomorphic to each other via the Poisson integral.

Proposition 2.9. Let $\varphi$ satisfy Assumption $(\varphi)$ and $u$ be a harmonic function on $\mathbb{R}_{+}^{n+1}$. Then $u \in H_{\varphi, 2}\left(\mathbb{R}_{+}^{n+1}\right)$ if and only if there exists $f \in H_{\varphi}\left(\mathbb{R}^{n}\right)$ such that, for all $(x, t) \in$ $\mathbb{R}_{+}^{n+1}, u(x, t)=\left(f * P_{t}\right)(x)$, where $\left(f * P_{t}\right)(x)$ is defined as in Remark 2.5. Moreover, there exists a positive constant $C$, independent of $f$ and $u$, such that

$$
\frac{1}{C}\|f\|_{H_{\varphi}\left(\mathbb{R}^{n}\right)} \leq\|u\|_{H_{\varphi}\left(\mathbb{R}_{+}^{n+1}\right)} \leq C\|f\|_{H_{\varphi}\left(\mathbb{R}^{n}\right)} .
$$


Proof. By Definition 2.7 and Remark 1.3(iii), to prove Proposition 2.9, it suffices to show that the Poisson integral $P_{t}$ is an isomorphism from $\left(H_{\varphi}\left(\mathbb{R}^{n}\right) \cap L^{2}\left(\mathbb{R}^{n}\right),\|\cdot\|_{H_{\varphi}\left(\mathbb{R}^{n}\right)}\right)$ to $\left(H_{\varphi}\left(\mathbb{R}_{+}^{n+1}\right) \cap H^{2}\left(\mathbb{R}_{+}^{n+1}\right),\|\cdot\|_{H_{\varphi}\left(\mathbb{R}_{+}^{n+1}\right)}\right)$. Recall that the Poisson integral is an isomorphism from $L^{2}\left(\mathbb{R}^{n}\right)$ to $H^{2}\left(\mathbb{R}_{+}^{n+1}\right)$ (see, for example, [2, Theorem 7.17]).

The inclusion that

$$
P_{t}\left(H_{\varphi}\left(\mathbb{R}^{n}\right) \cap L^{2}\left(\mathbb{R}^{n}\right),\|\cdot\|_{H_{\varphi}\left(\mathbb{R}^{n}\right)}\right) \subset\left(H_{\varphi}\left(\mathbb{R}_{+}^{n+1}\right) \cap H^{2}\left(\mathbb{R}_{+}^{n+1}\right),\|\cdot\|_{H_{\varphi}\left(\mathbb{R}_{+}^{n+1}\right)}\right)
$$

is an easy consequence of Proposition 2.4, the details being omitted.

We now turn to the inverse inclusion. Let $u \in H_{\varphi}\left(\mathbb{R}_{+}^{n+1}\right) \cap H^{2}\left(\mathbb{R}_{+}^{n+1}\right)$. For any $x \in \mathbb{R}^{n}$ and $\epsilon \in(0, \infty)$, let $u_{\epsilon}(x, t):=u(x, t+\epsilon)$. Since $u \in H^{2}\left(\mathbb{R}_{+}^{n+1}\right)$, we know that $u_{\epsilon}$ can be represented as a Poisson integral: $u_{\epsilon}(x, t)=\left(f_{\epsilon} * P_{t}\right)(x)$ for all $(x, t) \in \mathbb{R}_{+}^{n+1}$, where $f_{\epsilon}(x):=u(x, \epsilon)$. Moreover, from Proposition 2.4 and the definition of the non-tangential maximal function, it follows that

$$
\sup _{\epsilon \in(0, \infty)}\left\|f_{\epsilon}\right\|_{H_{\varphi}\left(\mathbb{R}^{n}\right)} \sim \sup _{\epsilon \in(0, \infty)}\left\|\left(f_{\epsilon} * P_{t}\right)^{*}\right\|_{L^{\varphi}\left(\mathbb{R}^{n}\right)} \sim \sup _{\epsilon \in(0, \infty)}\left\|u_{\epsilon}^{*}\right\|_{L^{\varphi}\left(\mathbb{R}^{n}\right)} \lesssim\left\|u^{*}\right\|_{L^{\varphi}\left(\mathbb{R}^{n}\right)}
$$

Thus, $\left\{f_{\epsilon}\right\}_{\epsilon \in(0, \infty)}$ is a bounded set in $H_{\varphi}\left(\mathbb{R}^{n}\right)$ and hence in $\mathcal{S}^{\prime}\left(\mathbb{R}^{n}\right)$ (see 21, Proposition 5.1]). By the weak compactness of $\mathcal{S}^{\prime}\left(\mathbb{R}^{n}\right)$ (see, for example, [32, p. 119]), we conclude that there exist an $f \in \mathcal{S}^{\prime}\left(\mathbb{R}^{n}\right)$ and a subsequence $\left\{f_{k}\right\}_{k \in \mathbb{N}}$ such that $\left\{f_{k}\right\}_{k \in \mathbb{N}}$ converges weakly to $f$ in $\mathcal{S}^{\prime}\left(\mathbb{R}^{n}\right)$. This, together with (2.13), implies that, for all $(x, t) \in \mathbb{R}_{+}^{n+1}$,

$$
\lim _{k \rightarrow \infty}\left(f_{k} * P_{t}\right)(x)=\left(f * P_{t}\right)(x)=u(x, t) .
$$

Thus, by Proposition 2.4, Fatou's lemma and (2.15), we conclude that

$$
\begin{aligned}
\|f\|_{H_{\varphi}\left(\mathbb{R}^{n}\right)} & \sim\left\|\lim _{k \rightarrow \infty}\left(f_{k}\right)_{P}^{*}\right\|_{L^{\varphi}\left(\mathbb{R}^{n}\right)} \lesssim \varliminf_{k \rightarrow \infty}\left\|\left(f_{k}\right)_{P}^{*}\right\|_{L^{\varphi}\left(\mathbb{R}^{n}\right)} \\
& \sim \varliminf_{k \rightarrow \infty}\left\|f_{k}\right\|_{H_{\varphi}\left(\mathbb{R}^{n}\right)} \lesssim\left\|u^{*}\right\|_{L^{\varphi}\left(\mathbb{R}^{n}\right)} \sim\|u\|_{H_{\varphi}\left(\mathbb{R}_{+}^{n+1}\right)}
\end{aligned}
$$

which immediately implies that $f \in H_{\varphi}\left(\mathbb{R}^{n}\right), u(x, t)=f * P_{t}(x)$ and hence completes the proof of Proposition 2.9.

\subsection{Musielak-Orlicz-Hardy spaces $\mathcal{H}_{\varphi}\left(\mathbb{R}_{+}^{n+1}\right)$ of harmonic vectors}

In this subsection, we study the Musielak-Orlicz-Hardy space $\mathcal{H}_{\varphi}\left(\mathbb{R}_{+}^{n+1}\right)$ consisting of vectors of harmonic functions which satisfy the so-called generalized Cauchy-Riemann equation. To be precise, let $F:=\left\{u_{0}, u_{1}, \ldots, u_{n}\right\}$ be a harmonic vector on $\mathbb{R}_{+}^{n+1}$. Then $F$ is said to satisfy the generalized Cauchy-Riemann equation, if, for all $j, k \in\{0, \ldots, n\}$,

$$
\left\{\begin{array}{l}
\sum_{j=0}^{n} \frac{\partial u_{j}}{\partial x_{j}}=0 \\
\frac{\partial u_{j}}{\partial x_{k}}=\frac{\partial u_{k}}{\partial x_{j}}
\end{array}\right.
$$

where, for $(x, t) \in \mathbb{R}_{+}^{n+1}$, we let $x:=\left(x_{1}, \ldots, x_{n}\right)$ and $x_{0}:=t$. 
Definition 2.10. Let $\varphi$ be a Musielak-Orlicz function satisfying Assumption $(\varphi)$. The Musielak-Orlicz-Hardy space $\mathcal{H}_{\varphi}\left(\mathbb{R}_{+}^{n+1}\right)$ of harmonic vectors is defined to be the space of all harmonic vectors $F:=\left\{u_{0}, u_{1}, \ldots, u_{n}\right\}$ on $\mathbb{R}_{+}^{n+1}$ satisfying (2.16) such that, for all $t \in(0, \infty)$

$$
|F(\cdot, t)|:=\left\{\sum_{j=0}^{n}\left|u_{j}(\cdot, t)\right|^{2}\right\}^{1 / 2} \in L^{\varphi}\left(\mathbb{R}^{n}\right) .
$$

Moreover, for any $F \in \mathcal{H}_{\varphi}\left(\mathbb{R}_{+}^{n+1}\right)$, its quasi-norm is defined by setting,

$$
\|F\|_{\mathcal{H}_{\varphi}\left(\mathbb{R}_{+}^{n+1}\right)}:=\sup _{t \in(0, \infty)}\||F(\cdot, t)|\|_{L^{\varphi}\left(\mathbb{R}^{n}\right)} .
$$

For $p \in(1, \infty)$, the Musielak-Orlicz-Hardy space $\mathcal{H}^{p}\left(\mathbb{R}_{+}^{n+1}\right)$ of harmonic vectors is defined as $\mathcal{H}_{\varphi}\left(\mathbb{R}_{+}^{n+1}\right)$ with $L^{\varphi}\left(\mathbb{R}^{n}\right)$ replaced by $L^{p}\left(\mathbb{R}^{n}\right)$. In particular, for any $F \in \mathcal{H}^{p}\left(\mathbb{R}_{+}^{n+1}\right)$, its norm is defined by setting,

$$
\|F\|_{\mathcal{H}^{p}\left(\mathbb{R}_{+}^{n+1}\right)}:=\sup _{t \in(0, \infty)}\||F(\cdot, t)|\|_{L^{p}\left(\mathbb{R}^{n}\right)} .
$$

Moreover, let

$$
\mathcal{H}_{\varphi, 2}\left(\mathbb{R}_{+}^{n+1}\right):=\overline{\mathcal{H}_{\varphi}\left(\mathbb{R}_{+}^{n+1}\right) \cap \mathcal{H}^{2}\left(\mathbb{R}_{+}^{n+1}\right)}\|\cdot\|_{\mathcal{H} \varphi\left(\mathbb{R}_{+}^{n+1}\right)}
$$

be the completion of the set $\mathcal{H}_{\varphi}\left(\mathbb{R}_{+}^{n+1}\right) \cap \mathcal{H}^{2}\left(\mathbb{R}_{+}^{n+1}\right)$ under the quasi-norm $\|\cdot\|_{\mathcal{H}_{\varphi}\left(\mathbb{R}_{+}^{n+1}\right)}$.

Remark 2.11. The space $\mathcal{H}^{p}\left(\mathbb{R}_{+}^{n+1}\right)$ was first introduced by Stein and Weiss to give a higher dimensional generalization of the Hardy space on the upper plane (see [33, 34, 35] for more details).

For any $F \in \mathcal{H}_{\varphi}\left(\mathbb{R}_{+}^{n+1}\right)$, we have the following technical lemmas, respectively, on the harmonic majorant and the boundary value of $F$.

Lemma 2.12. Assume that the function $\varphi$ satisfies Assumption $(\varphi)$ with $\frac{i(\varphi)}{q(\varphi)}>\frac{n-1}{n}$ and $F:=\left\{u_{0}, u_{1}, \ldots, u_{n}\right\} \in \mathcal{H}_{\varphi}\left(\mathbb{R}_{+}^{n+1}\right)$, where $i(\varphi)$ and $q(\varphi)$ are as in (1.5) and (1.6), respectively. Then, for all $q \in\left[\frac{n-1}{n}, \frac{i(\varphi)}{q(\varphi)}\right), a \in(0, \infty)$ and $(x, t) \in \mathbb{R}_{+}^{n+1}$,

$$
|F(x, t+a)|^{q} \leq\left(|F(x, a)|^{q} * P_{t}\right)(x),
$$

where $P_{t}$ is the Poisson kernel as in (2.12).

Proof. For all $t \in[0, \infty)$, let

$$
K\left(|F|^{q}, t\right):=\int_{\mathbb{R}^{n}} \frac{|F(x, t)|^{q}}{(|x|+1+t)^{n+1}} d x .
$$

Since $|F|^{q}$ is subharmonic on $\mathbb{R}_{+}^{n+1}$ (see [31, p. 234, Theorem 4.14]), by [28, p. 245, Theorem $2]$, in order to prove (2.17), it suffices to show that

$$
\lim _{t \rightarrow \infty} K\left(|F|^{q}, t\right)=0
$$


We now prove (2.18). Write

$$
\begin{aligned}
K\left(|F|^{q}, t\right) & =\int_{\left\{x \in \mathbb{R}^{n}:|F(x, t)| \geq 1\right\}} \frac{|F(x, t)|^{q}}{(|x|+1+t)^{n+1}} d x+\int_{\left\{x \in \mathbb{R}^{n}:|F(x, t)|<1\right\}} \cdots \\
& =: \mathrm{I}+\mathrm{II} .
\end{aligned}
$$

We first estimate I. By choosing $r \in(q(\varphi), \infty)$ satisfying $r<\frac{i(\varphi) n}{n-1}$ and $\frac{n-1}{n} \leq q<\frac{i(\varphi)}{r}$, we know that, for all $(x, t) \in \mathbb{R}_{+}^{n+1}, \varphi(\cdot, t) \in A_{r}\left(\mathbb{R}^{n}\right)$ and $\varphi(x, \cdot)$ is of lower type $q r$, which, together with Hölder's inequality, further implies that

$$
\begin{aligned}
\mathrm{I} & \lesssim\left\{\int_{\left\{x \in \mathbb{R}^{n}:|F(x, t)| \geq 1\right\}}|F(x, t)|^{q r} \varphi(x, 1) d x\right\}^{\frac{1}{r}} \\
& \times\left\{\int_{\left\{x \in \mathbb{R}^{n}:|F(x, t)| \geq 1\right\}} \frac{1}{(|x|+1+t)^{(n+1) r^{\prime}}}[\varphi(x, 1)]^{-r^{\prime} / r} d x\right\}^{\frac{1}{r^{\prime}}} \\
\lesssim & \left\{\int_{\left\{x \in \mathbb{R}^{n}:|F(x, t)| \geq 1\right\}} \varphi(x,|F(x, t)|) d x\right\}^{1 / r} \\
& \times\left\{\int_{\left\{x \in \mathbb{R}^{n}:|F(x, t)| \geq 1\right\}} \frac{1}{(|x|+1+t)^{(n+1) r^{\prime}}}[\varphi(x, 1)]^{-r^{\prime} / r} d x\right\}^{1 / r^{\prime}} .
\end{aligned}
$$

Since $\varphi(\cdot, 1) \in A_{r}\left(\mathbb{R}^{n}\right)$, we see $w(\cdot):=[\varphi(\cdot, 1)]^{-r^{\prime} / r} \in A_{r^{\prime}}\left(\mathbb{R}^{n}\right)$ (see, for example, [12, p. 394, Theorem 1.14(c)]), which, together with [18, Lemma 1], implies that $w$ satisfies the so-called $B_{r^{\prime}}\left(\mathbb{R}^{n}\right)$-condition, namely, for all $x \in \mathbb{R}^{n}$,

$$
\int_{\mathbb{R}^{n}} \frac{w(y)}{(t+|x-y|)^{n r^{\prime}}} d y \lesssim t^{-n r^{\prime}} \int_{B(x, t)} w(y) d y
$$

By this, together with (2.20), we further see that

$$
\begin{aligned}
\mathrm{I} & \lesssim \frac{1}{1+t}\left\{\int_{\mathbb{R}^{n}} \varphi(x,|F(x, t)|) d x\right\}^{1 / r}\left\{\int_{\mathbb{R}^{n}} \frac{[\varphi(x, 1)]^{-r^{\prime} / r}}{(|x|+1+t)^{n r^{\prime}}} d x\right\}^{1 / r^{\prime}} \\
& \leq C_{(\varphi), 1} \frac{1}{1+t},
\end{aligned}
$$

where $C_{(\varphi), 1}$ is a positive constant, depending on $\varphi$, but independent of $t$.

To estimate the term II, let $\widetilde{r}:=\frac{1}{q}$. It is easy to see that $r<\frac{i(\varphi)}{q} \leq \frac{1}{q}=\widetilde{r}$. Thus, $\varphi(\cdot, 1) \in A_{\widetilde{r}}\left(\mathbb{R}^{n}\right)$, which, together with Hölder's inequality, the upper type 1 property of $\varphi(x, \cdot)$ and $(2.21)$, implies that

$$
\begin{aligned}
\mathrm{II} \lesssim & \left\{\int_{\mathbb{R}^{n}}|F(x, t)|^{q \widetilde{r}} \varphi(x, 1) d x\right\}^{1 / \widetilde{r}} \\
& \times\left\{\int_{B(0,1)} \frac{1}{(|x|+1+t)^{(n+1) \widetilde{r}^{\prime}}}[\varphi(x, 1)]^{-\widetilde{r}^{\prime} / \widetilde{r}} d x\right\}^{1 / \widetilde{r}^{\prime}}
\end{aligned}
$$




$$
\begin{aligned}
& \lesssim \frac{1}{1+t}\left\{\int_{\mathbb{R}^{n}} \varphi(x,|F(x, t)|) d x\right\}^{1 / \widetilde{r}}\left\{\int_{B(0,1)}[\varphi(x, 1)]^{-\widetilde{r}^{\prime} / \widetilde{r}} d x\right\}^{1 / \widetilde{r}^{\prime}} \\
& \leq C_{(\varphi), 2} \frac{1}{1+t}
\end{aligned}
$$

where $C_{(\varphi), 2}$ is a positive constant, depending on $\varphi$, but independent of $t$. Combining (2.19), (2.22) and (2.23), we see that (2.18) holds true. This finishes the proof of Lemma 2.12 ,

Lemma 2.13. Assume that the function $\varphi$ satisfies Assumption $(\varphi)$ with $\frac{i(\varphi)}{q(\varphi)}>\frac{n-1}{n}$ and $F:=\left\{u_{0}, u_{1}, \ldots, u_{n}\right\} \in \mathcal{H}_{\varphi}\left(\mathbb{R}_{+}^{n+1}\right)$, where $i(\varphi)$ and $q(\varphi)$ are as in (1.5) and (1.6), respectively. Then there exists $h \in L^{\varphi}\left(\mathbb{R}^{n}\right)$ such that $\lim _{t \rightarrow 0}|F(\cdot, t)|=h(\cdot)$ in $L^{\varphi}\left(\mathbb{R}^{n}\right)$ and $h$ is the non-tangential limit of $F$ as $t \rightarrow 0$ almost everywhere, namely, for almost every $x_{0} \in \mathbb{R}^{n}, \lim _{(x, t) \rightarrow\left(x_{0}, 0^{+}\right)}|F(x, t)|=h\left(x_{0}\right)$ for all $(x, t)$ in the cone $\Gamma\left(x_{0}\right):=\{(x, t) \in$ $\left.\mathbb{R}_{+}^{n+1}:\left|x-x_{0}\right|<t\right\}$. Moreover, for all $q \in\left[\frac{n-1}{n}, \frac{i(\varphi)}{q(\varphi)}\right)$ and $(x, t) \in \mathbb{R}_{+}^{n+1}$,

$$
|F(x, t)| \leq\left[\left(h^{q} * P_{t}\right)(x)\right]^{1 / q},
$$

where $P_{t}$ is the Poisson kernel as in (2.12).

Proof. For $F \in \mathcal{H}_{\varphi}\left(\mathbb{R}_{+}^{n+1}\right)$ and all $(x, t) \in \mathbb{R}_{+}^{n+1}$, let

$$
F_{1}(x, t):=\chi_{\left\{(x, t) \in \mathbb{R}_{+}^{n+1}:|F(x, t)| \geq 1\right\}} F(x, t)
$$

and

$$
F_{2}(x, t):=\chi_{\left\{(x, t) \in \mathbb{R}_{+}^{n+1}:|F(x, t)|<1\right\}} F(x, t) .
$$

Let $r \in(q(\varphi), \infty)$ satisfy $q<\frac{i(\varphi)}{r}$. Then, by the lower type $q r$ property of $\varphi(x, \cdot)$, we know that

$$
\begin{aligned}
\sup _{t \in(0, \infty)}\left\|\left|F_{1}(\cdot, t)\right|^{q}\right\|_{L_{\varphi(\cdot, 1)}^{r}}^{r}\left(\mathbb{R}^{n}\right) & =\sup _{t \in(0, \infty)}\left\{\int_{\mathbb{R}^{n}}\left|F_{1}(x, t)\right|^{q r} \varphi(x, 1) d x\right\} \\
& \leq \sup _{t \in(0, \infty)}\left\{\int_{\mathbb{R}^{n}} \varphi\left(x,\left|F_{1}(x, t)\right|\right) d x\right\} \\
& \leq \sup _{t \in(0, \infty)}\left\{\int_{\mathbb{R}^{n}} \varphi(x,|F(x, t)|) d x\right\}<\infty
\end{aligned}
$$

Thus, $\left\{\left|F_{1}(\cdot, t)\right|^{q}\right\}_{t>0}$ is uniformly bounded in $L_{\varphi(\cdot, 1)}^{r}\left(\mathbb{R}^{n}\right)$, which, together with the weak compactness of $L_{\varphi(\cdot, 1)}^{r}\left(\mathbb{R}^{n}\right)$, implies that there exist $\widetilde{h}_{1} \in L_{\varphi(\cdot, 1)}^{r}\left(\mathbb{R}^{n}\right)$ and a subsequence $\left\{\left|F_{1}\left(\cdot, t_{k}\right)\right|^{q}\right\}_{k \in \mathbb{N}}$ such that $t_{k} \rightarrow 0^{+}$and $\left\{\left|F_{1}\left(\cdot, t_{k}\right)\right|^{q}\right\}_{k \in \mathbb{N}}$ converges weakly to $\widetilde{h}_{1}$ in $L_{\varphi(\cdot, 1)}^{r}\left(\mathbb{R}^{n}\right)$ as $k \rightarrow \infty$, namely, for any $g \in L_{\varphi(\cdot, 1)}^{r^{\prime}}\left(\mathbb{R}^{n}\right)$,

$$
\lim _{k \rightarrow \infty} \int_{\mathbb{R}^{n}}\left|F_{1}\left(y, t_{k}\right)\right|^{q} g(y) \varphi(y, 1) d y=\int_{\mathbb{R}^{n}} \widetilde{h}_{1}(y) g(y) \varphi(y, 1) d y .
$$


Now, for all $y \in \mathbb{R}^{n}$, let

$$
g(y):=\frac{P_{t}(x-y)}{\varphi(y, 1)}
$$

where $P_{t}$ is the Poisson kernel as in (2.12). By using the $B_{r^{\prime}}\left(\mathbb{R}^{n}\right)$-condition as in (2.21) and $\varphi(\cdot, 1) \in A_{r}\left(\mathbb{R}^{n}\right)$, we conclude that

$$
\begin{aligned}
\int_{\mathbb{R}^{n}}|g(y)|^{r^{\prime}} \varphi(y, 1) d y & =\int_{\mathbb{R}^{n}}\left[\frac{P_{t}(x-y)}{\varphi(y, 1)}\right]^{r^{\prime}} \varphi(y, 1) d y \\
& \lesssim \int_{\mathbb{R}^{n}} \frac{1}{(t+|x-y|)^{n r^{\prime}}}[\varphi(y, 1)]^{-r^{\prime} / r} d y \\
& \lesssim \int_{B(x, t)}[\varphi(y, 1)]^{-r^{\prime} / r} d y<\infty,
\end{aligned}
$$

which implies that $g \in L_{\varphi(\cdot, 1)}^{r^{\prime}}\left(\mathbb{R}^{n}\right)$. Thus, from (2.25), we deduce that, for all $(x, t) \in$ $\mathbb{R}_{+}^{n+1}$

$$
\lim _{k \rightarrow \infty}\left(\left|F_{1}\left(\cdot, t_{k}\right)\right|^{q} * P_{t}\right)(x)=\left(\widetilde{h}_{1} * P_{t}\right)(x) .
$$

On the other hand, since $\sup _{(x, t) \in \mathbb{R}_{+}^{n+1}}\left|F_{2}(x, t)\right|^{q} \leq 1$, we know that $\left\{\left|F_{2}(\cdot, t)\right|^{q}\right\}_{t>0}$ is uniformly bounded in $L_{\varphi(\cdot, 1)}^{\infty}\left(\mathbb{R}^{n}\right)$. Thus, there exist $\widetilde{h}_{2} \in L_{\varphi(\cdot, 1)}^{\infty}\left(\mathbb{R}^{n}\right)$ with $\left\|\widetilde{h}_{2}\right\|_{L_{\varphi(\cdot, 1)}^{\infty}\left(\mathbb{R}^{n}\right)} \leq$ 1 and a subsequence $\left\{\left|F_{2}\left(\cdot, t_{k}\right)\right|^{q}\right\}_{k \in \mathbb{N}}$ such that $t_{k} \rightarrow 0^{+}$and $\left\{\left|F_{2}\left(\cdot, t_{k}\right)\right|^{q}\right\}_{k \in \mathbb{N}}$ converges *-weakly to $\widetilde{h}_{2}$ in $L_{\varphi(\cdot, 1)}^{\infty}\left(\mathbb{R}^{n}\right)$ as $k \rightarrow \infty$, namely, for any $g \in L_{\varphi(\cdot, 1)}^{1}\left(\mathbb{R}^{n}\right)$,

$$
\lim _{k \rightarrow \infty} \int_{\mathbb{R}^{n}}\left|F_{2}\left(y, t_{k}\right)\right|^{q} g(y) \varphi(y, 1) d y=\int_{\mathbb{R}^{n}} \widetilde{h}_{2}(y) g(y) \varphi(y, 1) d y .
$$

Here, by abuse of notation, we use the same subscripts for the above two different subsequences in our arguments.

Let $g$ be as in (2.26). It is easy to see that $\int_{\mathbb{R}^{n}} g(y) \varphi(y, 1) d y=1$. Thus, by (2.28), we find that, for all $x \in \mathbb{R}^{n}$,

$$
\lim _{k \rightarrow \infty}\left(\left|F_{2}\left(\cdot, t_{k}\right)\right|^{q} * P_{t}\right)(x)=\left(\widetilde{h}_{2} * P_{t}\right)(x) .
$$

Now, let $\widetilde{h}:=\widetilde{h}_{1}+\widetilde{h}_{2}$. Observe that, for all $k \in \mathbb{N},\left|\operatorname{supp}\left(F_{1}\left(\cdot, t_{k}\right)\right) \cap \operatorname{supp}\left(F_{2}\left(\cdot, t_{k}\right)\right)\right|=$ 0 , which further implies that $\left|\operatorname{supp}\left(\widetilde{h}_{1}\right) \cap \operatorname{supp}\left(\widetilde{h}_{2}\right)\right|=0$. Moreover, from (2.27) and (2.29), it follows that, for all $x \in \mathbb{R}^{n}$,

$$
\lim _{k \rightarrow \infty}\left(\left|F\left(\cdot, t_{k}\right)\right|^{q} * P_{t}\right)(x)=\left(\widetilde{h} * P_{t}\right)(x) .
$$

This, together with $t_{k} \rightarrow 0^{+}$as $k \rightarrow \infty$ and Lemma 2.12, shows that, for all $(x, t) \in \mathbb{R}_{+}^{n+1}$,

$$
|F(x, t)|^{q}=\lim _{k \rightarrow \infty}\left|F\left(x, t+t_{k}\right)\right|^{q} \leq \lim _{k \rightarrow \infty}\left(\left|F\left(\cdot, t_{k}\right)\right|^{q} * P_{t}\right)(x)=\left(\widetilde{h} * P_{t}\right)(x),
$$


which proves (2.24) by taking $h:=\widetilde{h}^{1 / q}$.

Now, we prove that $\widetilde{h}$ is the non-tangential limit of $\left|F\left(\cdot, t_{k}\right)\right|^{q}$. Using (2.27) and (2.29), we conclude that, for all $x \in \mathbb{R}^{n}$,

$$
\begin{aligned}
|F|^{*}(x) & \lesssim\left[\sup _{|y-x|<t, t \in(0, \infty)}\left(\widetilde{h}_{1} * P_{t}\right)(y)\right]^{1 / q}+\left[\sup _{|y-x|<t, t \in(0, \infty)}\left(\widetilde{h}_{2} * P_{t}\right)(y)\right]^{1 / q} \\
& =: \mathrm{I}+\mathrm{II} .
\end{aligned}
$$

To estimate II, from the fact that $\left\|\widetilde{h}_{2}\right\|_{L_{\varphi(\cdot, 1)}^{\infty}\left(\mathbb{R}^{n}\right)} \leq 1$ and (2.1), we deduce that $\left\|\widetilde{h}_{2}\right\|_{L^{\infty}\left(\mathbb{R}^{n}\right)} \leq 1$. This implies that

$$
\mathrm{II} \lesssim\left[\sup _{|y-x|<t, t \in(0, \infty)} \int_{\mathbb{R}^{n}} P_{t}(y) d y\right]^{1 / q} \lesssim 1 .
$$

For I, it is easy to see that

$$
\mathrm{I} \sim\left[\left(\widetilde{h}_{1}\right)_{P}^{*}(x)\right]^{1 / q} .
$$

Moreover, by the fact that $\varphi(\cdot, 1) \in A_{r}\left(\mathbb{R}^{n}\right), r \in(q(\varphi), \infty)$ and the boundedness of the Hardy-Littlewood maximal function $\mathcal{M}$ on $L_{\varphi(\cdot, 1)}^{r}\left(\mathbb{R}^{n}\right)$ (see, for example, [14, Theorem 9.1.9]), we conclude that

$$
\begin{aligned}
\int_{\mathbb{R}^{n}}\left[\left(\widetilde{h}_{1}\right)_{P}^{*}(x)\right]^{r} \varphi(x, 1) d x & \lesssim \int_{\mathbb{R}^{n}}\left[\mathcal{M}\left(\widetilde{h}_{1}\right)(x)\right]^{r} \varphi(x, 1) d x \\
& \lesssim \int_{\mathbb{R}^{n}}\left[\widetilde{h}_{1}(x)\right]^{r} \varphi(x, 1) d x<\infty,
\end{aligned}
$$

which, together with (2.31), (2.32) and (2.33), implies that, for almost every $x \in \mathbb{R}^{n}$, $|F|^{*}(x)<\infty$. From the fact that each coordinate function of $F$ is harmonic on $\mathbb{R}_{+}^{n+1}$ and Fatou's theorem (see [31, p. 47]), we deduce that $F(x, t)$ has a non-tangential limit as $t \rightarrow 0^{+}$, which, combined with the uniqueness of the limit, implies that $\widetilde{h}$ is the nontangential limit of $|F(\cdot, t)|^{q}$ as $t \rightarrow 0^{+}$.

Now, we show that $h \in L^{\varphi}\left(\mathbb{R}^{n}\right)$ by using some properties of convex Musielak-Orlicz spaces from [7]. For all $(x, t) \in \mathbb{R}_{+}^{n+1}$, let

$$
\varphi_{q}(x, t):=\varphi\left(x, t^{1 / q}\right) .
$$

By an elementary calculation, we see that

$$
\||F(\cdot, t)|\|_{L^{\varphi}\left(\mathbb{R}^{n}\right)}=|||F(\cdot, t)|^{q} \|_{L^{\varphi q}\left(\mathbb{R}^{n}\right)}^{1 / q},
$$

which, together with the fact that $F \in \mathcal{H}_{\varphi}\left(\mathbb{R}_{+}^{n+1}\right)$, implies that $\left\{|F(\cdot, t)|^{q}\right\}_{t \in(0, \infty)}$ is uniformly bounded in $L^{\varphi_{q}}\left(\mathbb{R}^{n}\right)$.

Moreover, using the fact that $i\left(\varphi_{q}\right)=\frac{i(\varphi)}{q}>q(\varphi) \geq 1$ and

$$
\varphi_{q}(x, t) \sim \int_{0}^{t} \frac{\varphi_{q}(x, s)}{s} d s,
$$

we conclude that $\widetilde{\varphi}_{q}(x, s):=\varphi_{q}(x, s) / s$ satisfies the following properties: 
(i) $\lim _{s \rightarrow 0^{+}} \widetilde{\varphi}_{q}(x, s)=0, \lim _{t \rightarrow \infty} \widetilde{\varphi}_{q}(x, s)=\infty$ and, when $s \in(0, \infty), \widetilde{\varphi}_{q}(x, s)>0$;

(ii) for all $x \in \mathbb{R}^{n}, \widetilde{\varphi}_{q}(x, \cdot)$ is decreasing;

(iii) for all $x \in \mathbb{R}^{n}, \widetilde{\varphi}_{q}(x, \cdot)$ is right continuous.

Thus, from [1, p. 262], we deduce that, for all $x \in \mathbb{R}^{n}, \varphi_{q}(x, \cdot)$ is equivalent to an $N$ function (see [1] for the definition of $N$-functions). Hence, by [7, p. 38, Theorem 2.3.13], we know that $L^{\varphi_{q}}\left(\mathbb{R}^{n}\right)$ is a Banach space.

For all $(x, t) \in \mathbb{R}_{+}^{n+1}$, let

$$
\varphi_{q}^{*}(x, t):=\sup _{s \in(0, \infty)}\left\{s t-\varphi_{q}(x, s)\right\}
$$

It follows, from [7, p. 59], that $L^{\varphi_{q}}\left(\mathbb{R}^{n}\right) \subset\left(L^{\varphi_{q}^{*}}\left(\mathbb{R}^{n}\right)\right)^{*}$. Thus, by Alaoglu's theorem, we obtain the *-weak compactness of $L^{\varphi_{q}}\left(\mathbb{R}^{n}\right)$, which, together with the fact $\left\{|F(\cdot, t)|^{q}\right\}_{t \in(0, \infty)}$ is uniformly bounded in $L^{\varphi_{q}}\left(\mathbb{R}^{n}\right)$, implies that there exist $g \in L^{\varphi_{q}}\left(\mathbb{R}^{n}\right)$ and a subsequence $\left\{\left|F\left(\cdot, t_{k}\right)\right|^{q}\right\}_{k \in \mathbb{N}}$ such that $t_{k} \rightarrow 0^{+}$and $\left\{\left|F\left(\cdot, t_{k}\right)\right|^{q}\right\}_{k \in \mathbb{N}}$ converges $*$-weakly to $g$ in $L^{\varphi_{q}}\left(\mathbb{R}^{n}\right)$ as $k \rightarrow \infty$. Moreover, from the uniqueness of the limit, we deduce that, for almost every $x \in \mathbb{R}^{n}, h(x)=[g(x)]^{1 / q}$. Thus, $h \in L^{\varphi}\left(\mathbb{R}^{n}\right)$.

The formula, $\lim _{t \rightarrow 0^{+}}|F(\cdot, t)|=h(\cdot)$ in $L^{\varphi}\left(\mathbb{R}^{n}\right)$, follows immediately from the facts that $h$ is the non-tangential limit of $F$ as $t \rightarrow 0^{+}$almost everywhere, $F \in \mathcal{H}_{\varphi}\left(\mathbb{R}_{+}^{n+1}\right)$, $h \in L^{\varphi}\left(\mathbb{R}^{n}\right)$ and the dominated convergence theorem. This finishes the proof of Lemma 2.13 .

Remark 2.14. We point out that, in the proofs of Lemmas 2.12 and 2.13 , we used the condition $\frac{i(\varphi)}{q(\varphi)}>\frac{n-1}{n}$ merely because we need the fact that $|F|^{q}$ is subharmonic on $\mathbb{R}_{+}^{n+1}$ for $q \in\left[\frac{n-1}{n}, \infty\right)$. Thus, if there exists $q$, whose size is strictly less than $\frac{n-1}{n}$, such that $|F|^{q}$ is subharmonic on $\mathbb{R}_{+}^{n+1}$, then, for all $\frac{i(\varphi)}{q(\varphi)}>q$, the conclusions of Lemmas 2.12 and 2.13 still hold true. Moreover, if, for all $q \in(0, \infty),|F|^{q}$ is subharmonic on $\mathbb{R}_{+}^{n+1}$, then, for every Musielak-Orlicz function $\varphi$ satisfying Assumption $(\varphi)$, by taking $q$ sufficiently small, we see that $\frac{i(\varphi)}{q(\varphi)}>q$ always holds true. Thus, in this case, Lemmas 2.12 and 2.13 hold true for every Musielak-Orlicz function satisfying Assumption $(\varphi)$.

With these preparations, we now turn to the study of the relation between $\mathcal{H}_{\varphi}\left(\mathbb{R}_{+}^{n+1}\right)$ and $H_{\varphi}\left(\mathbb{R}_{+}^{n+1}\right)$.

Proposition 2.15. Let $\varphi$ satisfy Assumption $(\varphi)$ with $\frac{i(\varphi)}{q(\varphi)}>\frac{n-1}{n}$ and

$$
F:=\left\{u_{0}, u_{1}, \ldots, u_{n}\right\} \in \mathcal{H}_{\varphi}\left(\mathbb{R}_{+}^{n+1}\right)
$$

where $i(\varphi)$ and $q(\varphi)$ are as in (1.5) and (1.6), respectively. Then there exists a harmonic function $u:=u_{0} \in H_{\varphi}\left(\mathbb{R}_{+}^{n+1}\right)$ such that

$$
\|u\|_{H_{\varphi}\left(\mathbb{R}_{+}^{n+1}\right)} \leq C\|F\|_{\mathcal{H}_{\varphi}\left(\mathbb{R}_{+}^{n+1}\right)}
$$

where $C$ is a positive constant independent of $u$ and $F$. 
Proof. Let $F \in \mathcal{H}_{\varphi}\left(\mathbb{R}_{+}^{n+1}\right)$. By Lemmas 2.12 and 2.13, we see that $|F|$ has the nontangential limit $F(\cdot, 0)$. Moreover, for all $(x, t) \in \mathbb{R}_{+}^{n+1}$,

$$
|F(x, t)|^{q} \leq\left(|F(\cdot, 0)|^{q} * P_{t}\right)(x) \lesssim \mathcal{M}\left(|F(\cdot, 0)|^{q}\right)(x),
$$

where $q \in\left[\frac{n-1}{n}, \frac{i(\varphi)}{q(\varphi)}\right)$ is as in Lemma 2.13 and $\mathcal{M}$ denotes the Hardy-Littlewood maximal function. Let $u:=u_{0}$ and $\varphi_{q}$ be as in (2.34). For all $\lambda \in(0, \infty)$, from (2.37), the fact that $q(\varphi)<\frac{i(\varphi)}{q}$ and Lemma 2.3, it follows that

$$
\begin{aligned}
\int_{\mathbb{R}^{n}} \varphi\left(x, \frac{u^{*}(x)}{\lambda}\right) d x & \leq \int_{\mathbb{R}^{n}} \varphi_{q}\left(x, \frac{\left(|F|^{q}\right)^{*}(x)}{\lambda^{q}}\right) d x \\
& \lesssim \int_{\mathbb{R}^{n}} \varphi_{q}\left(x, \frac{\left(\mathcal{M}\left(|F(\cdot, 0)|^{q}\right)\right)^{*}(x)}{\lambda^{q}}\right) d x \\
& \lesssim \int_{\mathbb{R}^{n}} \varphi_{q}\left(x, \frac{|F(x, 0)|^{q}}{\lambda^{q}}\right) d x \\
& \sim \int_{\mathbb{R}^{n}} \varphi\left(x, \frac{|F(x, 0)|}{\lambda}\right) d x \lesssim \sup _{t \in(0, \infty)} \int_{\mathbb{R}^{n}} \varphi\left(x, \frac{|F(x, t)|}{\lambda}\right) d x,
\end{aligned}
$$

which immediately implies (2.36) and hence completes the proof of Proposition 2.15.

Proposition 2.15 immediately implies the following conclusion, the details being omitted.

Corollary 2.16. Let $\varphi$ satisfy Assumption $(\varphi)$ with $\frac{i(\varphi)}{q(\varphi)}>\frac{n-1}{n}$ and

$$
F:=\left\{u_{0}, u_{1}, \ldots, u_{n}\right\} \in \mathcal{H}_{\varphi, 2}\left(\mathbb{R}_{+}^{n+1}\right)
$$

where $i(\varphi)$ and $q(\varphi)$ are as in (1.5) and (1.6), respectively. Then there exists a harmonic function $u:=u_{0} \in H_{\varphi, 2}\left(\mathbb{R}_{+}^{n+1}\right)$ such that

$$
\|u\|_{H_{\varphi}\left(\mathbb{R}_{+}^{n+1}\right)} \leq C\|F\|_{\mathcal{H}_{\varphi}\left(\mathbb{R}_{+}^{n+1}\right)}
$$

where $C$ is a positive constant independent of $u$ and $F$.

Furthermore, we have the following relation between $H_{\varphi}\left(\mathbb{R}^{n}\right)$ and $\mathcal{H}_{\varphi, 2}\left(\mathbb{R}_{+}^{n+1}\right)$, which also implies that $H_{\varphi}\left(\mathbb{R}^{n}\right)$ consists of the boundary values of real parts of $\mathcal{H}_{\varphi, 2}\left(\mathbb{R}_{+}^{n+1}\right)$.

Proposition 2.17. Let $\varphi$ satisfy Assumption $(\varphi)$ and $f \in H_{\varphi}\left(\mathbb{R}^{n}\right)$. Then there exists $F:=$ $\left\{u_{0}, u_{1}, \ldots, u_{n}\right\} \in \mathcal{H}_{\varphi, 2}\left(\mathbb{R}_{+}^{n+1}\right)$ such that $F$ satisfies the generalized Cauchy-Riemann equation (2.16) and that, for all $(x, t) \in \mathbb{R}_{+}^{n+1}, u_{0}(x, t):=\left(f * P_{t}\right)(x)$, where $P_{t}$ is the Poisson kernel as in (2.12). Moreover,

$$
\|F\|_{\mathcal{H}_{\varphi}\left(\mathbb{R}_{+}^{n+1}\right)} \leq C\|f\|_{H_{\varphi}\left(\mathbb{R}^{n}\right)},
$$

where $C$ is a positive constant independent of $f$ and $F$. 
Proof. Let $f \in H_{\varphi}\left(\mathbb{R}^{n}\right)$. By Remark 1.3(iii), we see that $L^{2}\left(\mathbb{R}^{n}\right) \cap H_{\varphi}\left(\mathbb{R}^{n}\right)$ is dense in $H_{\varphi}\left(\mathbb{R}^{n}\right)$. Thus, there exists a sequence $\left\{f_{k}\right\}_{k \in \mathbb{N}} \subset\left(L^{2}\left(\mathbb{R}^{n}\right) \cap H_{\varphi}\left(\mathbb{R}^{n}\right)\right)$ such that $\lim _{k \rightarrow \infty} f_{k}=f$ in $H_{\varphi}\left(\mathbb{R}^{n}\right)$ and hence in $\mathcal{S}^{\prime}\left(\mathbb{R}^{n}\right)$.

For any $k \in \mathbb{N}, j \in\{1, \ldots, n\}$ and $(x, t) \in \mathbb{R}_{+}^{n+1}$, let $u_{0}^{k}(x, t):=\left(f_{k} * P_{t}\right)(x)$ and $u_{j}^{k}(x, t):=\left(f_{k} * Q_{t}^{(j)}\right)(x)$, where $P_{t}$ is the Poisson kernel as in (2.12) and $Q_{t}^{(j)}$ the $j$-th conjugate Poisson kernel defined by setting, for all $x \in \mathbb{R}^{n}$,

$$
Q_{t}^{(j)}(x):=C_{(n)} \frac{x_{j}}{\left(t^{2}+|x|^{2}\right)^{\frac{n+1}{2}}}
$$

where $C_{(n)}$ is as in (1.1).

Since $f_{k} \in L^{2}\left(\mathbb{R}^{n}\right)$, we deduce, from [35, p. 236, Theorem 4.17], that the harmonic vector $F_{k}:=\left\{u_{0}^{k}, u_{1}^{k}, \ldots, u_{n}^{k}\right\} \in \mathcal{H}^{2}\left(\mathbb{R}_{+}^{n+1}\right)$ and satisfies the generalized Cauchy-Riemann equation (2.16). Moreover, by using the Fourier transform, we see that, for all $j \in\{0,1, \ldots, n\}$ and $(x, t) \in \mathbb{R}_{+}^{n+1},\left(Q_{t}^{(j)} * f_{k}\right)(x)=\left(R_{j}\left(f_{k}\right) * P_{t}\right)(x)$ (see also [31, p. 65, Theorem 3]), which, together with Proposition 2.4 and the boundedness of $R_{j}$ on $H_{\varphi}\left(\mathbb{R}^{n}\right)$ (see Corollary 2.22 below), implies that, for all $j \in\{0,1, \ldots, n\}$,

$$
\sup _{t \in(0, \infty)}\left\||| u_{j}^{k}(\cdot, t) \mid\right\|_{L^{\varphi}\left(\mathbb{R}^{n}\right)} \lesssim\left\|R_{j}\left(f_{k}\right)\right\|_{H_{\varphi}\left(\mathbb{R}^{n}\right)} \lesssim\left\|f_{k}\right\|_{H_{\varphi}\left(\mathbb{R}^{n}\right)} \lesssim\|f\|_{H_{\varphi}\left(\mathbb{R}^{n}\right)} .
$$

Thus,

$$
\sup _{t \in(0, \infty)}\left\|F_{k}(\cdot, t)\right\|_{L^{\varphi}\left(\mathbb{R}^{n}\right)} \lesssim\|f\|_{H_{\varphi}\left(\mathbb{R}^{n}\right)}<\infty
$$

which implies that $F_{k} \in \mathcal{H}_{\varphi}\left(\mathbb{R}_{+}^{n+1}\right)$ and hence $F_{k} \in \mathcal{H}_{\varphi}\left(\mathbb{R}_{+}^{n+1}\right) \cap \mathcal{H}^{2}\left(\mathbb{R}_{+}^{n+1}\right)$.

We point out that, in the above argument, we used the boundedness of the Riesz transform $R_{j}$ on $H_{\varphi}\left(\mathbb{R}^{n}\right)$, which will be proved in Corollary 2.22 below, whose proof does not use the conclusion of Proposition 2.17. So, there exists no risk of circular reasoning.

On the other hand, from $\lim _{k \rightarrow \infty} f_{k}=f$ in $H_{\varphi}\left(\mathbb{R}^{n}\right)$ and hence in $\mathcal{S}^{\prime}\left(\mathbb{R}^{n}\right)$,

$$
\lim _{k \rightarrow \infty} R_{j}\left(f_{k}\right)=R_{j}(f)
$$

in $H_{\varphi}\left(\mathbb{R}^{n}\right)$ and hence in $\mathcal{S}^{\prime}\left(\mathbb{R}^{n}\right)$, and (2.13) , we deduce that, for all $(x, t) \in \mathbb{R}_{+}^{n+1}$, $\lim _{k \rightarrow \infty} f_{k} * P_{t}(x)=f * P_{t}(x)$ and $\lim _{k \rightarrow \infty} R_{j}\left(f_{k}\right) * P_{t}(x)=R_{j}(f) * P_{t}(x)$.

Now, we claim that the above two limits are uniform on compact sets. Indeed, for all $(x, t) \in \mathbb{R}_{+}^{n+1}, y, z \in B\left(x, \frac{t}{4}\right), \widetilde{t} \in\left(\frac{3 t}{4}, \frac{5 t}{4}\right)$ and $\phi \in \mathcal{S}\left(\mathbb{R}^{n}\right)$ satisfies $\int_{\mathbb{R}^{n}} \phi(x) d x=1$, by the definition of the non-tangential maximal function, we know that

$$
\left|\left(\left[f_{k}-f\right] * \phi_{\widetilde{t}}\right)(z)\right| \leq \mathcal{M}_{\phi}^{*}\left(f_{k}-f\right)(y) .
$$

Moreover, for any $\epsilon \in(0,1]$ and $q \in(I(\varphi), \infty)$, from the upper type $q$ property of $\varphi(x, \cdot)$, it follows that

$$
\epsilon^{q} \int_{\left\{x \in \mathbb{R}^{n}: \mathcal{M}_{\phi}^{*}\left(f_{k}-f\right)(x)>\epsilon\right\}} \varphi(x, 1) d x \lesssim \int_{\left\{x \in \mathbb{R}^{n}: \mathcal{M}_{\phi}^{*}\left(f_{k}-f\right)(x)>\epsilon\right\}} \varphi(x, \epsilon) d x
$$




$$
\lesssim \int_{\mathbb{R}^{n}} \varphi\left(x, \mathcal{M}_{\phi}^{*}\left(f_{k}-f\right)(x)\right) d x
$$

which tends to 0 as $k \rightarrow \infty$. Thus, $\mathcal{M}_{\phi}^{*}\left(f_{k}-f\right)$ converges to 0 in the measure $\varphi(\cdot, 1) d x$. This shows that there exists $k_{0} \in \mathbb{N}$ such that, for all $k \in \mathbb{N}$ with $k \geq k_{0}$,

$$
\int_{B\left(x, \frac{t}{4}\right)} \varphi(y, 1) d y \geq 2 \int_{\mathrm{E}_{k}^{\complement}} \varphi(y, 1) d y,
$$

where $\mathrm{E}_{k}:=\left\{y \in B\left(x, \frac{t}{4}\right): \mathcal{M}_{\phi}^{*}\left(f_{k}-f\right)(y)<1\right\}$.

Combined (2.41) with (2.42) and the upper type 1 property of $\varphi(x, \cdot)$, we conclude that, for all $z \in B\left(x, \frac{t}{4}\right)$ and $\widetilde{t} \in\left(\frac{3 t}{4}, \frac{5 t}{4}\right)$,

$$
\begin{aligned}
\left|\left(\left[f_{k}-f\right] * \phi_{\widetilde{t}}\right)(z)\right| & \leq \frac{1}{\int_{\mathrm{E}_{k}} \varphi(y, 1) d y} \int_{\mathrm{E}_{k}} \mathcal{M}_{\phi}^{*}\left(f_{k}-f\right)(y) \varphi(y, 1) d y \\
& \lesssim \frac{1}{\int_{B\left(x, \frac{t}{4}\right)} \varphi(y, 1) d y} \int_{\mathrm{E}_{k}} \varphi\left(y, \mathcal{M}_{\phi}^{*}\left(f_{k}-f\right)(y)\right) d y
\end{aligned}
$$

which tends to 0 as $k \rightarrow \infty$. This implies that $f_{k} * \phi_{t}$ converges uniformly to $f * \phi_{t}$ on $B\left(x, \frac{t}{4}\right) \times\left(\frac{3 t}{4}, \frac{5 t}{4}\right)$.

Moreover, using (2.13), we know $\lim _{k \rightarrow \infty} f_{k} * P_{t}(x)=f * P_{t}(x)$ uniform on compact sets. Similarly, we also conclude $\lim _{k \rightarrow \infty} R_{j}\left(f_{k}\right) * P_{t}(x)=R_{j}(f) * P_{t}(x)$ uniform on compact sets. This shows the above claim.

By the above claim and the fact that $F_{k}$ satisfies the generalized Cauchy-Riemann equation (2.16), we know that $F:=\left\{f * P_{t}, R_{1}(f) * P_{t}, \ldots, R_{n}(f) * P_{t}\right\}$ also satisfies the generalized Cauchy-Riemann equation (2.16), which, together with Fatou's lemma and (2.40), implies that

$$
\begin{aligned}
\sup _{t \in(0, \infty)}\||F(\cdot, t)|\|_{L^{\varphi}\left(\mathbb{R}^{n}\right)} & =\sup _{t \in(0, \infty)}\left\|\lim _{k \rightarrow \infty}\left|F_{k}(\cdot, t)\right|\right\|_{L^{\varphi}\left(\mathbb{R}^{n}\right)} \\
& \leq \sup _{t \in(0, \infty)} \underset{k \rightarrow \infty}{\lim }\left\|\left|F_{k}(\cdot, t)\right|\right\|_{L^{\varphi}\left(\mathbb{R}^{n}\right)} \lesssim\|f\|_{H_{\varphi}\left(\mathbb{R}^{n}\right)}<\infty .
\end{aligned}
$$

Thus, $F \in \mathcal{H}_{\varphi, 2}\left(\mathbb{R}_{+}^{n+1}\right)$ and (2.38) holds true, which completes the proof of Proposition 2.17 ,

Combined Propositions 2.9, 2.15 with 2.17, we immediately obtain the following conclusion.

Theorem 2.18. Let $\varphi$ satisfy Assumption $(\varphi)$ with $\frac{i(\varphi)}{q(\varphi)}>\frac{n-1}{n}$, where $i(\varphi)$ and $q(\varphi)$ are as in (1.5) and (1.6), respectively. Then the spaces $H_{\varphi}\left(\mathbb{R}^{n}\right), H_{\varphi, 2}\left(\mathbb{R}_{+}^{n+1}\right)$ and $\mathcal{H}_{\varphi, 2}\left(\mathbb{R}_{+}^{n+1}\right)$, defined, respectively, in Definitions 1.2, 2.7 and 2.10, are isomorphic to each other.

More precisely, the following statements hold true:

(i) $u \in H_{\varphi, 2}\left(\mathbb{R}_{+}^{n+1}\right)$ if and only if there exists $f \in H_{\varphi}\left(\mathbb{R}^{n}\right)$ such that, for all $(x, t) \in$ $\mathbb{R}_{+}^{n+1}, u(x, t)=\left(f * P_{t}\right)(x)$, where $P_{t}$ is the Poisson kernel as in (2.12).

(ii) If $F:=\left(u_{0}, u_{1}, \ldots, u_{n}\right) \in \mathcal{H}_{\varphi, 2}\left(\mathbb{R}_{+}^{n+1}\right)$, then $u_{0} \in H_{\varphi, 2}\left(\mathbb{R}_{+}^{n+1}\right)$.

(iii) If $f \in H_{\varphi}\left(\mathbb{R}^{n}\right)$, then there exists $F:=\left\{u_{0}, u_{1}, \ldots, u_{n}\right\} \in \mathcal{H}_{\varphi, 2}\left(\mathbb{R}_{+}^{n+1}\right)$ such that, for all $(x, t) \in \mathbb{R}_{+}^{n+1}, u_{0}(x, t):=f * P_{t}(x)$. 


\subsection{First order Riesz transform characterizations}

In this subsection, we give out the proof of Theorem 1.5. To this end, we first give a sufficient condition on operators to be bounded on $H_{\varphi}\left(\mathbb{R}^{n}\right)$. We now recall the notion of $H_{\varphi}\left(\mathbb{R}^{n}\right)$-atoms introduced in [23, Definition 2.4] as follows.

Definition 2.19 ([23]). Let $\varphi$ satisfy Assumption $(\varphi), q \in(q(\varphi), \infty]$ and $s \in \mathbb{Z}_{+}$satisfy $s \geq\left\lfloor n\left[\frac{q(\varphi)}{i(\varphi)}-1\right]\right\rfloor$, where $q(\varphi)$ and $i(\varphi)$ are as in (1.6) and (1.5), respectively. A measurable function $a$ on $\mathbb{R}^{n}$ is called a $(\varphi, q, s)$-atom, if there exists a ball $B \subset \mathbb{R}^{n}$ such that

(i) $\operatorname{supp} a \subset B$;

(ii) $\|a\|_{L_{\varphi}^{q}(B)} \leq\left\|\chi_{B}\right\|_{L^{\varphi}\left(\mathbb{R}^{n}\right)}^{-1}$, where

$$
\|a\|_{L_{\varphi}^{q}(B)}:= \begin{cases}\sup _{t \in(0, \infty)}\left[\frac{1}{\varphi(B, t)} \int_{\mathbb{R}^{n}}|a(x)|^{q} \varphi(x, t) d x\right]^{1 / q}, & q \in[1, \infty), \\ \|a\|_{L^{\infty}(B)}, & q=\infty,\end{cases}
$$

and $\varphi(B, t):=\int_{B} \varphi(x, t) d x$

(iii) $\int_{\mathbb{R}^{n}} a(x) x^{\alpha} d x=0$ for all $\alpha:=\left(\alpha_{1}, \ldots, \alpha_{n}\right) \in \mathbb{Z}_{+}^{n}$ with $|\alpha|:=\alpha_{1}+\cdots+\alpha_{n} \leq s$.

Let $T$ be a sublinear operator. Recall that $T$ is said to be nonnegative if, for all $f$ in the domain of $T, T f \geq 0$.

Lemma 2.20. Let $\varphi$ satisfy Assumption $(\varphi)$ and $s \in \mathbb{Z}_{+}$satisfy $s \geq m(\varphi):=\left\lfloor n\left(\frac{q(\varphi)}{i(\varphi)}-1\right)\right\rfloor$, where $i(\varphi)$ and $q(\varphi)$ are as in (1.5) and (1.6), respectively. Suppose that $T$ is a linear (resp. nonnegative sublinear) operator, which is of weak type $\left(L^{2}\left(\mathbb{R}^{n}\right), L^{2}\left(\mathbb{R}^{n}\right)\right)$. If there exists a positive constant $C$ such that, for every $\lambda \in \mathbb{C}$ and $(\varphi, q, s)$-atom a associated with the ball $B$,

$$
\int_{\mathbb{R}^{n}} \varphi(x, T(\lambda a)(x)) d x \leq C \int_{B} \varphi\left(x, \frac{|\lambda|}{\left\|\chi_{B}\right\|_{L^{\varphi}\left(\mathbb{R}^{n}\right)}}\right) d x,
$$

then $T$ can be extended to a bounded linear (resp. nonnegative sublinear) operator from $H_{\varphi}\left(\mathbb{R}^{n}\right)$ to $L^{\varphi}\left(\mathbb{R}^{n}\right)$.

Proof. Lemma 2.20 is a special case of [44, Lemma 5.6] when the operator $L$ considered therein is the Laplace operator $-\Delta$. The only difference is that here we use the $(\varphi, q, s)$ atoms to replace the operator-adapted atoms therein, the details being omitted. This finishes the proof of Lemma 2.20 .

Using Lemma 2.20, we establish the following proposition of the interpolation of operators.

Proposition 2.21. Let $\varphi$ satisfy Assumption $(\varphi), I(\varphi)$ and $i(\varphi)$ be as in (1.4) and (1.5), respectively. Assume that $T$ is a linear (resp. nonnegative sublinear) operator and either of the following two conditions holds true:

(i) if $0<p_{1}<i(\varphi) \leq I(\varphi) \leq 1<p_{2}<\infty$ and, for all $t \in(0, \infty)$, $T$ is of weak type $\left(H_{\varphi(\cdot, t)}^{p_{1}}\left(\mathbb{R}^{n}\right), L_{\varphi(\cdot, t)}^{p_{1}}\left(\mathbb{R}^{n}\right)\right)$ and of weak type $\left(L_{\varphi(\cdot, t)}^{p_{2}}\left(\mathbb{R}^{n}\right), L_{\varphi(\cdot, t)}^{p_{2}}\left(\mathbb{R}^{n}\right)\right) ;$ 
(ii) if $0<p_{1}<i(\varphi) \leq I(\varphi)<p_{2} \leq 1$ and, for all $t \in(0, \infty)$, $T$ is of weak type $\left(H_{\varphi(\cdot, t)}^{p_{1}}\left(\mathbb{R}^{n}\right), L_{\varphi(\cdot, t)}^{p_{1}}\left(\mathbb{R}^{n}\right)\right)$ and of weak type $\left(H_{\varphi(\cdot, t)}^{p_{2}}\left(\mathbb{R}^{n}\right), L_{\varphi(\cdot, t)}^{p_{2}}\left(\mathbb{R}^{n}\right)\right)$.

Then $T$ is bounded from $H_{\varphi}\left(\mathbb{R}^{n}\right)$ to $L^{\varphi}\left(\mathbb{R}^{n}\right)$.

Proof. Assume first that (i) holds true. Let $q \in\left(\max \left\{q(\varphi), p_{2}\right\}, \infty\right), s \in \mathbb{Z}_{+}$satisfy $s \geq\left\lfloor n\left(\frac{q(\varphi)}{p_{1}}-1\right)\right\rfloor$ with $q(\varphi)$ as in (1.6) $), \lambda \in(0, \infty)$ and $a$ be a $(\varphi, q, s)$-atom associated with the ball $B$. From the fact that $T$ is of weak type $\left(L_{\varphi(\cdot, t)}^{p_{2}}\left(\mathbb{R}^{n}\right), L_{\varphi(\cdot, t)}^{p_{2}}\left(\mathbb{R}^{n}\right)\right)$, Definition 2.19(ii) and Hölder's inequality, it follows that, for all $\alpha \in(0, \infty)$,

$$
\begin{aligned}
\int_{\left\{x \in \mathbb{R}^{n}:|T(\lambda a)(x)|>\alpha\right\}} \varphi(x, t) d x & \lesssim \frac{1}{\alpha^{p_{2}}} \int_{\mathbb{R}^{n}}|\lambda a(x)|^{p_{2}} \varphi(x, t) d x \\
& \sim \frac{\lambda^{p_{2}}}{\alpha^{p_{2}}}\left[\frac{1}{\varphi(B, t)} \int_{\mathbb{R}^{n}}|a(x)|^{p_{2}} \varphi(x, t) d x\right] \varphi(B, t) \\
& \lesssim \frac{\lambda^{p_{2}}}{\alpha^{p_{2}}}\left\|\chi_{B}\right\|_{L^{\varphi}\left(\mathbb{R}^{n}\right)}^{-p_{2}} \varphi(B, t) .
\end{aligned}
$$

On the other hand, by Definition 2.19 again, we conclude that

$$
\begin{aligned}
\|\| \chi_{B}\left\|_{L^{\varphi}\left(\mathbb{R}^{n}\right)}[\varphi(B, t)]^{-\frac{1}{p_{1}}} a\right\|_{L_{\varphi(\cdot, t)}^{q}\left(\mathbb{R}^{n}\right)} & =\left\|\chi_{B}\right\|_{L^{\varphi}\left(\mathbb{R}^{n}\right)}[\varphi(B, t)]^{\frac{1}{q}-\frac{1}{p_{1}}} \frac{\|a\|_{L_{\varphi(\cdot, t)}^{q}\left(\mathbb{R}^{n}\right)}}{[\varphi(B, t)]^{1 / q}} \\
& \leq[\varphi(B, t)]^{\frac{1}{q}-\frac{1}{p_{1}}}
\end{aligned}
$$

which immediately implies that $\left\|\chi_{B}\right\|_{L^{\varphi}\left(\mathbb{R}^{n}\right)}[\varphi(B, t)]^{-\frac{1}{p_{1}}} a$ is a weighted $\left(p_{1}, q, s\right)$-atom associated with $B$ (see [11, 37] for its definition). This, together with the fact that $T$ is of weak type $\left(H_{\varphi(\cdot, t)}^{p_{1}}\left(\mathbb{R}^{n}\right), L_{\varphi(\cdot, t)}^{p_{1}}\left(\mathbb{R}^{n}\right)\right)$, implies that

$$
\begin{aligned}
& \int_{\left\{x \in \mathbb{R}^{n}:|T(\lambda a)(x)|>\alpha\right\}} \varphi(x, t) d x \\
& \quad=\int_{\left\{x \in \mathbb{R}^{n}:\left|T\left(\lambda\left\|\chi_{B}\right\|_{L^{\varphi}\left(\mathbb{R}^{n}\right)}[\varphi(B, t)]^{-1 / p_{1}} a\right)(x)\right|>\alpha\left\|\chi_{B}\right\|_{L^{\varphi}\left(\mathbb{R}^{n}\right)}[\varphi(B, t)]^{\left.-1 / p_{1}\right\}}\right.} \varphi(x, t) d x \\
& \lesssim \frac{\lambda^{p_{1}}}{\alpha^{p_{1}}}\left\|\chi_{B}\right\|_{L^{\varphi}\left(\mathbb{R}^{n}\right)}^{-p_{1}} \varphi(B, t) .
\end{aligned}
$$

Now, let $R:=\frac{\lambda}{\left\|\chi_{B}\right\|_{L^{\varphi}\left(\mathbb{R}^{n}\right)}}$. From the fact that, for all $(x, t) \in \mathbb{R}^{n} \times(0, \infty), \varphi(x, t) \sim$ $\int_{0}^{t} \frac{\varphi(x, s)}{s} d s$ and Fubini's theorem, we deduce that

$$
\begin{aligned}
\int_{\mathbb{R}^{n}} \varphi(x, T(\lambda a)(x)) d x & \sim \int_{0}^{\infty} \frac{1}{t} \int_{\left\{x \in \mathbb{R}^{n}:|T(\lambda a)(x)|>t\right\}} \varphi(x, t) d x d t \\
& \sim \int_{0}^{R} \frac{1}{t} \int_{\left\{x \in \mathbb{R}^{n}:|T(\lambda a)(x)|>t\right\}} \varphi(x, t) d x d t+\int_{R}^{\infty} \cdots \\
& =: \text { I }+ \text { II. }
\end{aligned}
$$

For I, taking $\epsilon \in(0, \infty)$ sufficiently small so that $\frac{\varphi(x, t)}{t^{p_{1}+\epsilon}}$ is increasing in $t$, by using (2.45) and the fact $R=\frac{\lambda}{\left\|\chi_{B}\right\|_{L \varphi\left(\mathbb{R}^{n}\right)}}$, we see that

$$
\mathrm{I} \lesssim \int_{0}^{R} \frac{\lambda^{p_{1}}}{t^{1+p_{1}}}\left\|\chi_{B}\right\|_{L^{\varphi}\left(\mathbb{R}^{n}\right)}^{-p_{1}} \int_{B} \varphi(x, t) d x d t
$$




$$
\lesssim \int_{0}^{R} \frac{\lambda^{p_{1}}}{t^{1-\epsilon}} d t\left\|\chi_{B}\right\|_{L^{\varphi}\left(\mathbb{R}^{n}\right)}^{-p_{1}} \frac{1}{R^{p_{1}+\epsilon}} \int_{B} \varphi(x, R) d x \lesssim \int_{B} \varphi\left(x, \frac{\lambda}{\left\|\chi_{B}\right\|_{L^{\varphi}\left(\mathbb{R}^{n}\right)}}\right) d x .
$$

Similarly, choosing $\epsilon \in(0,1)$ sufficiently small such that $\frac{\varphi(x, t)}{t^{p^{2}-\epsilon}}$ is decreasing in $t$, it follows, from (2.44), that

$$
\mathrm{II} \lesssim \int_{R}^{\infty} \frac{\lambda^{p_{2}}}{t^{1+p_{2}}}\left\|\chi_{B}\right\|_{L^{\varphi}\left(\mathbb{R}^{n}\right)}^{-p_{2}} \int_{B} \varphi(x, t) d x d t \lesssim \int_{B} \varphi\left(x, \frac{\lambda}{\left\|\chi_{B}\right\|_{L^{\varphi}\left(\mathbb{R}^{n}\right)}}\right) d x,
$$

which, together with (2.46) and (2.47), implies that (2.43) of Lemma 2.20 holds true. This, combined with Lemma 2.20, finishes the proof of Proposition 2.21 when (i) holds true.

The proof of the case when (ii) holds true is similar, the details being omitted here. This finishes the proof of Proposition 2.21.

Corollary 2.22. Let $\varphi$ satisfy Assumption $(\varphi)$. Then, for all $j \in\{1, \ldots, n\}$, the Riesz transform $R_{j}$ is bounded on $H_{\varphi}\left(\mathbb{R}^{n}\right)$.

Proof. Let $\phi \in \mathcal{S}\left(\mathbb{R}^{n}\right)$ satisfy $\int_{\mathbb{R}^{n}} \phi(x) d x=1$. For all $j \in\{1, \ldots, n\}$, let $T_{j}:=\mathcal{M}_{\phi} \circ R_{j}$, where $\mathcal{M}_{\phi}$ is as in (2.4). Using Proposition 2.2 and the fact that, for all $p \in(0,1]$ and $w \in A_{\infty}\left(\mathbb{R}^{n}\right), R_{j}$ is bounded on the weighted Hardy space $H_{w}^{p}\left(\mathbb{R}^{n}\right)$ (see [21, Theorem 1.1]), we conclude that $T_{j}$ is bounded from $H_{w}^{p}\left(\mathbb{R}^{n}\right)$ to $L_{w}^{p}\left(\mathbb{R}^{n}\right)$. In particular, let $p_{1} \in(0, i(\varphi))$, since, for all $t \in(0, \infty), \varphi(\cdot, t) \in A_{\infty}\left(\mathbb{R}^{n}\right)$, we know that $T_{j}$ is bounded from $H_{\varphi(\cdot, t)}^{p_{1}}\left(\mathbb{R}^{n}\right)$ to $L_{\varphi(\cdot, t)}^{p_{1}}\left(\mathbb{R}^{n}\right)$.

On the other hand, let $q(\varphi)$ be as in (1.6) and $p_{2} \in(q(\varphi), \infty)$. From [12, p. 411, Theorem 3.1], we deduce that, for all $w \in A_{p_{2}}\left(\mathbb{R}^{n}\right), R_{j}$ is bounded on the weighted Lebesgue space $L_{w}^{p_{2}}\left(\mathbb{R}^{n}\right)$. Since $\varphi(\cdot, t) \in A_{p_{2}}\left(\mathbb{R}^{n}\right)$, we know $R_{j}$ is bounded on $L_{\varphi(\cdot, t)}^{p_{2}}\left(\mathbb{R}^{n}\right)$, which, together with the boundedness of $\mathcal{M}_{\phi}$ on $L_{\varphi(\cdot, t)}^{p_{2}}\left(\mathbb{R}^{n}\right)$, implies that $T_{j}$ is bounded on $L_{\varphi(\cdot, t)}^{p_{2}}\left(\mathbb{R}^{n}\right)$. Hence, using Propositions 2.2 and 2.21(i), we conclude

$$
\left\|R_{j}(f)\right\|_{H_{\varphi}\left(\mathbb{R}^{n}\right)} \sim\left\|T_{j}(f)\right\|_{L^{\varphi}\left(\mathbb{R}^{n}\right)} \lesssim\|f\|_{H_{\varphi}\left(\mathbb{R}^{n}\right)},
$$

which completes the proof of Corollary 2.22 ,

We point out that Proposition 2.21 can also be applied to the boundedness of CalderónZygmund operators on $H_{\varphi}\left(\mathbb{R}^{n}\right)$. Recall the following notion of $\theta$-Calderón-Zygmund operators from Yabuta [43]. Let $\theta$ be a nonnegative nondecreasing function on $(0, \infty)$ satisfying $\int_{0}^{1} \frac{\theta(t)}{t} d t<\infty$. A continuous function $K:\left(\mathbb{R}^{n} \times \mathbb{R}^{n}\right) \backslash\left\{(x, x): x \in \mathbb{R}^{n}\right\} \rightarrow \mathbb{C}$ is called a $\theta$ Calderón-Zygmund kernel, if there exists a positive constant $C$ such that, for all $x, y \in \mathbb{R}^{n}$ with $x \neq y$,

$$
|K(x, y)| \leq \frac{C}{|x-y|^{n}}
$$

and, for all $x, x^{\prime}, y \in \mathbb{R}^{n}$ with $2\left|x-x^{\prime}\right|<|x-y|$,

$$
\left|K(x, y)-K\left(x^{\prime}, y\right)\right|+\left|K(y, x)-K\left(y, x^{\prime}\right)\right| \leq \frac{C}{|x-y|^{n}} \theta\left(\frac{\left|x-x^{\prime}\right|}{|x-y|}\right) .
$$


A linear operator $T: \mathcal{S}\left(\mathbb{R}^{n}\right) \rightarrow \mathcal{S}^{\prime}\left(\mathbb{R}^{n}\right)$ is called a $\theta$-Calderón-Zygmund operator, if $\mathrm{T}$ can be extended to a bounded linear operator on $L^{2}\left(\mathbb{R}^{n}\right)$ and there exists a $\theta$-Calderón-Zygmund kernel $K$ such that, for all $f \in C_{c}^{\infty}\left(\mathbb{R}^{n}\right)$ and $x \notin \operatorname{supp} f$,

$$
T f(x)=\int_{\mathbb{R}^{n}} K(x, y) f(y) d y .
$$

Recall also that a nonnegative locally integrable function $w$ on $\mathbb{R}^{n}$ is said to satisfy the reverse Hölder condition for some $q \in(1, \infty)$, denoted by $w \in \mathrm{RH}_{q}\left(\mathbb{R}^{n}\right)$, if there exists a positive constant $C$ such that, for all balls $B \subset \mathbb{R}^{n}$,

$$
\left\{\frac{1}{|B|} \int_{B}[w(x)]^{q} d x\right\}^{1 / q} \leq \frac{C}{|B|} \int_{B} w(x) d x .
$$

Corollary 2.23. Let $\delta \in(0,1]$, the function $\varphi$ satisfy Assumption $(\varphi), q \in\left[1, \frac{i(\varphi)(n+\delta)}{n}\right)$, $r \in\left(\frac{n+\delta}{n+\delta-n q}, \infty\right)$ and, for all $t \in(0, \infty), \varphi(\cdot, t) \in A_{q}\left(\mathbb{R}^{n}\right) \cap \mathrm{RH}_{r}\left(\mathbb{R}^{n}\right)$, where $i(\varphi)$ and $q(\varphi)$ are as in (1.5) and (1.6), respectively. Assume also that $\theta$ is a nondecreasing function on $[0, \infty)$ satisfying $\int_{0}^{\infty} \frac{\theta(t)}{t^{1+\delta}} d t<\infty$. If $T$ is a $\theta$-Caldrón-Zygmund operator satisfying $T^{*} 1=0$, namely, for all $f \in L^{\infty}\left(\mathbb{R}^{n}\right)$ with compact support and $\int_{\mathbb{R}^{n}} f(x) d x=0$,

$$
\int_{\mathbb{R}^{n}} T f(x) d x=0
$$

then $T$ is bounded on $H_{\varphi}\left(\mathbb{R}^{n}\right)$.

Proof. To prove Corollary 2.23, recall, in [21, Theorem 1.2], that Ky proved that, for all $\delta \in(0,1], p_{1} \in\left(\frac{n}{n+\delta}, 1\right], q \in\left[1, \frac{p_{1}(n+\delta)}{n}\right), r \in\left(\frac{n+\delta}{n+\delta-n q}, \infty\right)$ and $w \in A_{q}\left(\mathbb{R}^{n}\right) \cap \mathrm{RH}_{r}\left(\mathbb{R}^{n}\right)$, the $\theta$-Calderón-Zygmund operator $T$, with $\theta$ satisfying the same assumptions as in this corollary, is bounded on the weighted Hardy space $H_{w}^{p_{1}}\left(\mathbb{R}^{n}\right)$, if $T^{*} 1=0$. In particular, let $p_{1} \in\left(\frac{n}{n+\delta}, i(\varphi)\right)$, we know $q \in\left[1, \frac{i(\varphi)(n+\delta)}{n}\right)$ and $r \in\left(\frac{n+\delta}{n+\delta-n q}, \infty\right)$. Thus, for all $t \in(0, \infty)$, $\varphi(\cdot, t) \in A_{q}\left(\mathbb{R}^{n}\right) \cap \mathrm{RH}_{r}\left(\mathbb{R}^{n}\right)$ and hence $T$ is bounded on $H_{\varphi(\cdot, t)}^{p_{1}}\left(\mathbb{R}^{n}\right)$, if $T^{*} 1=0$.

On the other hand, let $q(\varphi)$ be as in (1.6). From [43, Theorem 2.4], we deduce that, for all $p_{2} \in(q(\varphi), \infty)$ and $w \in A_{p_{2}}\left(\mathbb{R}^{n}\right), T$ is bounded on $L_{w}^{p_{2}}\left(\mathbb{R}^{n}\right)$. Since, $p_{2}>q(\varphi)$, we know that, for all $t \in(0, \infty), \varphi(\cdot, t) \in A_{p_{2}}\left(\mathbb{R}^{n}\right)$. Thus, $T$ is bounded on $L_{\varphi(\cdot, t)}^{p_{2}}\left(\mathbb{R}^{n}\right)$. Moreover, let $\mathcal{M}_{\phi}$ be as in (2.4) and $S:=\mathcal{M}_{\phi} \circ T$. Using Proposition 2.2 and the boundedness of $\mathcal{M}_{\phi}$ on $L_{\varphi(\cdot, t)}^{p_{2}}\left(\mathbb{R}^{n}\right)$, we conclude that, for all $t \in(0, \infty), S$ is bounded from $H_{\varphi(\cdot, t)}^{p_{1}}\left(\mathbb{R}^{n}\right)$ to $L_{\varphi(\cdot, t)}^{p_{1}}\left(\mathbb{R}^{n}\right)$ and bounded on $L_{\varphi(\cdot, t)}^{p_{2}}\left(\mathbb{R}^{n}\right)$. By Proposition 2.21)(i), we know that $S$ is bounded from $H_{\varphi}\left(\mathbb{R}^{n}\right)$ to $L^{\varphi}\left(\mathbb{R}^{n}\right)$. This, together with Proposition 2.2, implies that $T$ is bounded on $H_{\varphi}\left(\mathbb{R}^{n}\right)$, which completes the proof of Corollary 2.23.

Remark 2.24. We point out that it is well known that many important operators are bounded on weighted Hardy spaces. Thus, by using Proposition 2.21, we can obtain their corresponding boundedness on $H_{\varphi}\left(\mathbb{R}^{n}\right)$.

We now turn to the proof of Theorem 1.5 . 
Proof of Theorem 1.5. We prove Theorem 1.5 by showing that

$$
\left(H_{\varphi}\left(\mathbb{R}^{n}\right) \cap L^{2}\left(\mathbb{R}^{n}\right)\right)=\mathbb{H}_{\varphi, \text { Riesz }}\left(\mathbb{R}^{n}\right)
$$

with equivalent quasi-norms.

We first show the inclusion that $\left(H_{\varphi}\left(\mathbb{R}^{n}\right) \cap L^{2}\left(\mathbb{R}^{n}\right)\right) \subset \mathbb{H}_{\varphi, \text { Riesz }}\left(\mathbb{R}^{n}\right)$. Let $f \in H_{\varphi}\left(\mathbb{R}^{n}\right) \cap$ $L^{2}\left(\mathbb{R}^{n}\right)$ and $\phi \in \mathcal{S}\left(\mathbb{R}^{n}\right)$ satisfy (2.3) . By Proposition 2.2 and Corollary 2.22, we see that

$$
\begin{aligned}
\|f\|_{H_{\varphi}, \operatorname{Riesz}\left(\mathbb{R}^{n}\right)} & =\|f\|_{L^{\varphi}\left(\mathbb{R}^{n}\right)}+\sum_{j=1}^{n}\left\|R_{j}(f)\right\|_{L^{\varphi}\left(\mathbb{R}^{n}\right)} \\
& \leq\left\|\mathcal{M}_{\phi}(f)\right\|_{L^{\varphi}\left(\mathbb{R}^{n}\right)}+\sum_{j=1}^{n}\left\|\mathcal{M}_{\phi}\left(R_{j}(f)\right)\right\|_{L^{\varphi}\left(\mathbb{R}^{n}\right)} \\
& \sim\|f\|_{H_{\varphi}\left(\mathbb{R}^{n}\right)}+\sum_{j=1}^{n}\left\|R_{j}(f)\right\|_{H_{\varphi}\left(\mathbb{R}^{n}\right)} \lesssim\|f\|_{H_{\varphi}\left(\mathbb{R}^{n}\right)},
\end{aligned}
$$

where $\mathcal{M}_{\phi}$ denotes the radial maximal function as in (2.4). This implies that $f \in$ $\mathbb{H}_{\varphi, \text { Riesz }}\left(\mathbb{R}^{n}\right)$ and hence the inclusion $\left(H_{\varphi}\left(\mathbb{R}^{n}\right) \cap L^{2}\left(\mathbb{R}^{n}\right)\right) \subset \mathbb{H}_{\varphi, \text { Riesz }}\left(\mathbb{R}^{n}\right)$ holds true.

We now turn to the proof of the inclusion $\mathbb{H}_{\varphi, \text { Riesz }}\left(\mathbb{R}^{n}\right) \subset\left(H_{\varphi}\left(\mathbb{R}^{n}\right) \cap L^{2}\left(\mathbb{R}^{n}\right)\right)$. Let $f \in \mathbb{H}_{\varphi, \text { Riesz }}\left(\mathbb{R}^{n}\right)$. For all $(x, t) \in \mathbb{R}_{+}^{n+1}$, let

$$
\begin{aligned}
F(x, t) & :=\left(u_{0}(x, t), u_{1}(x, t), \ldots, u_{n}(x, t)\right) \\
& :=\left(\left(f * P_{t}\right)(x),\left(f * Q_{t}^{1}\right)(x), \ldots,\left(f * Q_{t}^{n}\right)(x)\right),
\end{aligned}
$$

where $P_{t}$ is the Poisson kernel as in (2.12) and, for all $j \in\{1, \ldots, n\}, Q_{t}^{(j)}$ is the conjugant Poisson kernel as in (2.39). From $f \in L^{2}\left(\mathbb{R}^{n}\right)$ and [16] (see also [31, p. 78, 4.4]), we deduce that $F$ satisfies the generalized Cauchy-Riemann equation (2.16). Thus, we know that, for $q \in\left[\frac{n-1}{n}, \frac{i(\varphi)}{q(\varphi)}\right),|F|^{q}$ is subharmonic (see, for example, [35, p. 234, Theorem 4.14]). Moreover, by [35, p. 80, Theorem 4.6], we obtain the following harmonic majorant that, for all $(x, t) \in \mathbb{R}_{+}^{n+1}$,

$$
|F(x, t)|^{q} \leq\left(|F(\cdot, 0)|^{q} * P_{t}\right)(x),
$$

where $F(\cdot, 0)=\left\{f, R_{1}(f), \ldots, R_{n}(f)\right\}$ via the Fourier transform. Thus, it follows, from (2.35) and Lemma 2.3, that

$$
\begin{aligned}
& \sup _{t \in(0, \infty)}\||F(\cdot, t)|\|_{L^{\varphi}\left(\mathbb{R}^{n}\right)}=\sup _{t \in(0, \infty)}\left\||F(\cdot, t)|^{q}\right\|_{L^{\varphi q}\left(\mathbb{R}^{n}\right)}^{1 / q} \leq \sup _{t \in(0, \infty)}\left\|\mathcal{M}\left(|F(\cdot, 0)|^{q}\right)\right\|_{L^{\varphi^{q}}\left(\mathbb{R}^{n}\right)}^{1 / q} \\
& \lesssim \sup _{t \in(0, \infty)}\||F(\cdot, 0)|\|_{L^{\varphi}\left(\mathbb{R}^{n}\right)} \lesssim\|f\|_{L^{\varphi}\left(\mathbb{R}^{n}\right)}+\sum_{j=1}^{n}\left\|R_{j}(f)\right\|_{L^{\varphi}\left(\mathbb{R}^{n}\right)} \\
& \sim\|f\|_{H_{\mathbb{H}} \varphi, \operatorname{Riesz}\left(\mathbb{R}^{n}\right)},
\end{aligned}
$$

where $\varphi_{q}$ is as in (2.34) and $\mathcal{M}$ the Hardy-Littlewood maximal function as in (2.6). Thus, $F \in \mathcal{H}_{\varphi}\left(\mathbb{R}_{+}^{n+1}\right)$ and

$$
\|F\|_{\mathcal{H}_{\varphi}\left(\mathbb{R}_{+}^{n+1}\right)} \lesssim\|f\|_{H_{\mathbb{H}_{\varphi}, \text { Riesz }}\left(\mathbb{R}^{n}\right)} .
$$


Moreover, from $f \in L^{2}\left(\mathbb{R}^{n}\right)$ and [35, Theorem 4.17(i)], we further deduce $F \in \mathcal{H}^{2}\left(\mathbb{R}_{+}^{n+1}\right)$, which, together with $F \in \mathcal{H}_{\varphi}\left(\mathbb{R}_{+}^{n+1}\right)$ and Theorem 2.18, further implies that $f \in H_{\varphi}\left(\mathbb{R}^{n}\right)$ and $\|f\|_{H_{\varphi}\left(\mathbb{R}^{n}\right)} \lesssim\|f\|_{H_{\mathbb{H}_{\varphi}, \text { Riesz }}\left(\mathbb{R}^{n}\right)}$. Thus, $f \in H_{\varphi}\left(\mathbb{R}^{n}\right) \cap L^{2}\left(\mathbb{R}^{n}\right)$, which completes the proof of Theorem 1.5.

\section{Higher order Riesz transform characterizations}

In this section, we give out the proofs of Theorems 1.7 and 1.11. First, we introduce the Musielak-Orlicz-Hardy spaces $\mathcal{H}_{\varphi, m}\left(\mathbb{R}_{+}^{n+1}\right)$ of tensor-valued functions of rank $m$ with $m \in \mathbb{N}$.

Let $n, m \in \mathbb{N}$ and $\left\{e_{0}, e_{1}, \ldots, e_{n}\right\}$ be an orthonormal basis of $\mathbb{R}^{n+1}$. The tensor product of $m$ copies of $\mathbb{R}^{n+1}$ is defined to be the set

$$
\bigotimes^{m} \mathbb{R}^{n+1}:=\left\{F:=\sum_{j_{1}, \ldots, j_{m}=0}^{n} F_{j_{1}, \ldots, j_{m}} e_{j_{1}} \otimes \cdots \otimes e_{j_{m}}: \quad F_{j_{1}, \ldots, j_{m}} \in \mathbb{C}\right\}
$$

where $e_{j_{1}} \otimes \cdots \otimes e_{j_{m}}$ denotes the tensor product of $e_{j_{1}}, \ldots, e_{j_{m}}$ and each $F \in \stackrel{m}{\otimes} \mathbb{R}^{n+1}$ is called a tensor of rank $m$.

Let $F: \mathbb{R}_{+}^{n+1} \rightarrow \stackrel{m}{\bigotimes} \mathbb{R}^{n+1}$ be a tensor-valued function of rank $m$ of the form that, for all $(x, t) \in \mathbb{R}_{+}^{n+1}$,

$$
F(x, t)=\sum_{j_{1}, \ldots, j_{m}=0}^{n} F_{j_{1}, \ldots, j_{m}}(x, t) e_{j_{1}} \otimes \cdots \otimes e_{j_{m}}
$$

with $F_{j_{1}, \ldots, j_{m}}(x, t) \in \mathbb{C}$. Then the tensor-valued function $F$ of rank $m$ is said to be symmetric, if, for any permutation $\sigma$ on $\{1, \ldots, m\}, j_{1}, \ldots, j_{m} \in\{0, \ldots, n\}$ and $(x, t) \in$ $\mathbb{R}_{+}^{n+1}$

$$
F_{j_{1}, \ldots, j_{m}}(x, t)=F_{j_{\sigma(1)}, \ldots, j_{\sigma(m)}}(x, t) .
$$

For $F$ being symmetric, $F$ is said to be of trace zero if, for all $j_{3}, \ldots, j_{m} \in\{0, \ldots, n\}$ and $(x, t) \in \mathbb{R}_{+}^{n+1}$,

$$
\sum_{j=0}^{n} F_{j, j, j_{3}, \ldots, j_{m}}(x, t) \equiv 0
$$

Let $F$ be as in (3.1). Its gradient $\nabla F: \mathbb{R}_{+}^{n+1} \rightarrow \stackrel{m+1}{\bigotimes}^{n+1}$ is a tensor-valued function of rank $m+1$ of the form that, for all $(x, t) \in \mathbb{R}_{+}^{n+1}$,

$$
\begin{aligned}
\nabla F(x, t) & =\sum_{j=0}^{n} \frac{\partial F}{\partial x_{j}}(x, t) \otimes e_{j} \\
& =\sum_{j=0}^{n} \sum_{j_{1}, \ldots, j_{m}=0}^{n} \frac{\partial F_{j_{1}, \ldots, j_{m}}}{\partial x_{j}}(x, t) e_{j_{1}} \otimes \cdots \otimes e_{j_{m}} \otimes e_{j}
\end{aligned}
$$


here and hereafter, we always let $x_{0}:=t$. A tensor-valued function $F$ is said to satisfy the generalized Cauchy-Riemann equation, if both $F$ and $\nabla F$ are symmetric and of trace zero. We point out that, if $m=1$, this definition of generalized Cauchy-Riemann equations is equivalent to the generalized Cauchy-Riemann equation as in (2.16). For more details on the generalized Cauchy-Riemann equation on tensor-valued functions, we refer the reader to $[34,29$.

The following is a generalization of Musielak-Orlicz-Hardy spaces $\mathcal{H}_{\varphi}\left(\mathbb{R}_{+}^{n+1}\right)$ of harmonic vectors defined in Definition 2.10.

Definition 3.1. Let $m \in \mathbb{N}$ and $\varphi$ satisfy Assumption $(\varphi)$. The Musielak-Orlicz-Hardy space $\mathcal{H}_{\varphi, m}\left(\mathbb{R}_{+}^{n+1}\right)$ of tensor-valued functions of rank $m$ is defined to be the set of all tensorvalued functions $F$, of rank $m$, satisfying the generalized Cauchy-Riemann equation. For any $F \in \mathcal{H}_{\varphi, m}\left(\mathbb{R}_{+}^{n+1}\right)$, its quasi-norm is defined by

$$
\|F\|_{\mathcal{H}_{\varphi, m}\left(\mathbb{R}_{+}^{n+1}\right)}:=\sup _{t>0}\||F(\cdot, t)|\|_{L^{\varphi}\left(\mathbb{R}^{n}\right)},
$$

where, for all $(x, t) \in \mathbb{R}_{+}^{n+1}$,

$$
|F(x, t)|:=\left\{\sum_{j_{1}, \ldots, j_{m}=0}^{n}\left|F_{j_{1}, \ldots, j_{m}}(x, t)\right|^{2}\right\}^{1 / 2} .
$$

Moreover, Stein and Weiss 34 proved the following result.

Proposition $3.2(34])$. Let $m \in \mathbb{N}$ and $F$ be a tensor-valued functions of rank $m$ satisfying the generalized Cauchy-Riemann equation. Then, for all $p \in\left[\frac{n-1}{n+m-1}, \infty\right),|F|^{p}$ is subharmonic on $\mathbb{R}_{+}^{n+1}$.

Recall also the following result from Calderón and Zygmund [6, Theorem 1].

Proposition 3.3 ([6]). Let $m \in \mathbb{N}$ and $u$ be a harmonic function on $\mathbb{R}_{+}^{n+1}$. For all $p \in\left[\frac{n-1}{n+m-1}, \infty\right),\left|\nabla^{m} u\right|^{p}$ is subharmonic. Here, for all $(x, t) \in \mathbb{R}_{+}^{n+1}$,

$$
\nabla^{m} u(x, t):=\left\{\partial^{\alpha} u(x, t)\right\}_{|\alpha|=m}
$$

with $\alpha:=\left\{\alpha_{0}, \ldots, \alpha_{n}\right\} \in \mathbb{Z}_{+}^{n+1},|\alpha|:=\sum_{j=0}^{n}\left|\alpha_{j}\right|, x_{0}:=t$ and $\partial^{\alpha}:=\left(\frac{\partial}{\partial x_{0}}\right)^{\alpha_{0}} \cdots\left(\frac{\partial}{\partial x_{n}}\right)^{\alpha_{n}}$.

It is known that every harmonic vector satisfying the generalized Cauchy-Riemann equation (2.16) is a gradient of a harmonic function on $\mathbb{R}_{+}^{n+1}$. A similar result still holds true for tensor-valued functions, which is the following proposition.

Proposition 3.4 ([35, 39]). Let $m \in \mathbb{N}$ with $m \geq 2, F$ be a tensor-valued function of rank $m$ satisfying that both $F$ and $\nabla F$ are symmetric, and $F$ is of trace zero. Then there exists a harmonic function $u$ on $\mathbb{R}_{+}^{n+1}$ such that $\nabla^{m} u=F$, namely, for all $\left\{j_{1}, \ldots, j_{m}\right\} \subset$ $\{0,1, \ldots, n\}$ and $(x, t) \in \mathbb{R}_{+}^{n+1}$,

$$
\frac{\partial}{\partial x_{j_{1}}} \cdots \frac{\partial}{\partial x_{j_{m}}} u(x, t)=F_{j_{1}, \cdots, j_{m}}(x, t)
$$


Remark 3.5. (i) Propositions 3.3 and 3.4 imply that, if $m \geq 2$, then the condition that $\nabla F$ has trace zero, in the generalized Cauchy-Riemann equation, can be removed to ensure that Proposition 3.2 still holds true.

(ii) We also point out that, in Proposition 2.15, Lemmas 2.12 and 2.13, and Corollary 2.18, we used the restriction that $\frac{i(\varphi)}{q(\varphi)}>\frac{n-1}{n}$, only because, for all $p \in\left[\frac{n-1}{n}, \infty\right)$, the $p$-power of the absolute value of the first-order gradient $|\nabla u|^{p}$ of a harmonic function on $\mathbb{R}_{+}^{n+1}$ is subharmonic. Since, for all $m \in \mathbb{N}$ and $p \in\left[\frac{n-1}{n+m-1}, \infty\right),\left|\nabla^{m} u\right|^{p}$ is subharmonic on $\mathbb{R}_{+}^{n+1}$, the restriction $\frac{i(\varphi)}{q(\varphi)}>\frac{n-1}{n}$ can be relaxed to $\frac{i(\varphi)}{q(\varphi)}>\frac{n-1}{n+m-1}$, with the MusielakOrlicz-Hardy space $\mathcal{H}_{\varphi}\left(\mathbb{R}_{+}^{n+1}\right)$ of harmonic vectors replaced by the Musielak-Orlicz-Hardy space $\mathcal{H}_{\varphi, m}\left(\mathbb{R}_{+}^{n+1}\right)$ of tensor-valued functions of rank $m$. Moreover, for any given MusielakOrlicz function $\varphi$ satisfying Assumption $(\varphi)$, by letting $m$ be sufficiently large, we know that Proposition 2.15, Lemmas 2.12 and 2.13, and Theorem 2.18 always hold true for $\frac{i(\varphi)}{q(\varphi)}>\frac{n-1}{n+m-1}$.

Now we give out the proof of Theorem 1.7

Proof of Theorem 1.7. The proof of Theorem 1.7 is similar to that of Theorem 1.5. In particular, the second inequality of (1.10) is an easy consequence of Proposition 2.2 and Corollary 2.22. Indeed, let $f \in H_{\varphi}\left(\mathbb{R}^{n}\right) \cap L^{2}\left(\mathbb{R}^{n}\right)$. By Proposition 2.2. Corollary 2.22 and an argument similar to that used in (2.49), we see that

$$
\|f\|_{L^{\varphi}\left(\mathbb{R}^{n}\right)}+\sum_{k=1}^{m} \sum_{j_{1}, \ldots, j_{k}=1}^{n}\left\|R_{j_{1}} \cdots R_{j_{k}}(f)\right\|_{L^{\varphi}\left(\mathbb{R}^{n}\right)} \lesssim\|f\|_{H_{\varphi}\left(\mathbb{R}^{n}\right)},
$$

which implies the second inequality of (1.10).

To prove the first inequality of (1.10), let $f \in L^{2}\left(\mathbb{R}^{n}\right)$ satisfy (1.9). We construct the tensor-valued function $F$ of rank $m$ by setting, for all $\left\{j_{1}, \ldots, j_{m}\right\} \subset\{0, \ldots, n\}$ and $(x, t) \in \mathbb{R}_{+}^{n+1}$,

$$
F_{j_{1}, \ldots, j_{m}}(x, t):=\left(\left(R_{j_{1}} \cdots R_{j_{m}}(f)\right) * P_{t}\right)(x),
$$

where $P_{t}$ is the Poisson kernel as in (2.12) and $R_{0}:=I$ denotes the identity operator. We know $F:=\sum_{j_{1}, \ldots, j_{m}=0}^{n} F_{j_{1}, j_{2}, \ldots, j_{m}} e_{j_{1}} \otimes \cdots \otimes e_{j_{m}}$ satisfies the generalized CauchyRiemann equation via the Fourier transform (see also the proof of [39, Lemma 17.1]). Also, a corresponding harmonic majorant holds true (see also [39, Lemma 17.2]), namely, for all $q \in\left[\frac{n-1}{n+m-1}, \frac{i(\varphi)}{q(\varphi)}\right)$ and $(x, t) \in \mathbb{R}_{+}^{n+1}$,

$$
|F(x, t)|^{q} \leq\left(|F(x, 0)|^{q} * P_{t}\right)(x),
$$

where $F(x, 0):=\left\{R_{j_{1}} \cdots R_{j_{m}}(f)(x)\right\}_{\left\{j_{1}, \ldots, j_{m}\right\} \subset\{0, \ldots, n\}}$, which, combined with (2.35), (1.9) and Lemma 2.3, implies that $F \in \mathcal{H}_{\varphi, m}\left(\mathbb{R}_{+}^{n+1}\right)$ and

$$
\begin{aligned}
\|F\|_{\mathcal{H}_{\varphi, m}\left(\mathbb{R}_{+}^{n+1}\right)} & =\sup _{t \in(0, \infty)}\|F(\cdot, t) \mid\|_{L^{\varphi}\left(\mathbb{R}^{n}\right)} \lesssim\left\|\mathcal{M}\left(|F(\cdot, 0)|^{q}\right)\right\|_{L^{\varphi q}\left(\mathbb{R}^{n}\right)}^{1 / q} \\
& \lesssim \sum_{j_{1}, \ldots, j_{m}=0}^{n}\left\|R_{j_{1}} \cdots R_{j_{m}}(f)\right\|_{L^{\varphi}\left(\mathbb{R}^{n}\right)}
\end{aligned}
$$




$$
\lesssim\|f\|_{L^{\varphi}\left(\mathbb{R}^{n}\right)}+\sum_{k=1}^{m} \sum_{j_{1}, \ldots, j_{k}=1}^{n}\left\|R_{j_{1}} \cdots R_{j_{k}}(f)\right\|_{L^{\varphi}\left(\mathbb{R}^{n}\right)} \lesssim A,
$$

where $\mathcal{M}$ denotes the Hardy-Littlewood maximal function as in (2.6). This, together with Remark 3.5(ii) (a counterpart to Theorem 2.18), implies that $f \in H_{\varphi}\left(\mathbb{R}^{n}\right)$ and the first inequality of (1.10) holds true, which completes the proof of Theorem 1.7.

We now turn to the proof of Theorem 1.11, To this end, we recall some facts on Fourier multipliers.

Let $f \in \mathcal{S}\left(\mathbb{R}^{n}\right), \mathbb{S}^{n-1}$ be the unit sphere in $\mathbb{R}^{n}$ and $\theta \in L^{\infty}\left(\mathbb{S}^{n-1}\right)$. The Fourier multiplier $K$ of $f$ with the multiplier function $\theta$ is defined by setting, for all $\xi \in \mathbb{R}^{n}$,

$$
K(f)(\xi):=\mathcal{F}^{-1}\left(\theta\left(\frac{\cdot}{|\cdot|}\right) \mathcal{F}(f)(\cdot)\right)(\xi)
$$

where $\mathcal{F}$ and $\mathcal{F}^{-1}$ denote, respectively, the Fourier transform and its inverse.

It is easy to see that, for all $j \in\{1, \ldots, n\}$, the Riesz transform $R_{j}$ is a Fourier multiplier with the multiplier function $\theta_{j}(\xi):=-i \xi_{j}$ for all $\xi \in \mathbb{S}^{n-1}$. Also, for all $k \in \mathbb{N}$ and $\left\{j_{1}, \ldots, j_{k}\right\} \subset\{1, \ldots, n\}$, the higher Riesz transform $R_{j_{1}} \cdots R_{j_{k}}$ is also a Fourier multiplier with the multiplier function that, for all $\xi \in \mathbb{S}^{n-1}$,

$$
\theta_{j_{1}, \ldots, j_{k}}(\xi):=\left(-i \xi_{j_{1}}\right) \cdots\left(-i \xi_{j_{k}}\right)
$$

Proposition 3.6. Let $\varphi$ satisfy Assumption $(\varphi)$ and $\theta \in C^{\infty}\left(\mathbb{S}^{n-1}\right)$. Then the Fourier multiplier $K$ with the multiplier function $\theta$ is bounded on $H_{\varphi}\left(\mathbb{R}^{n}\right)$.

Proof. By $\theta \in C^{\infty}\left(\mathbb{S}^{n-1}\right)$, we deduce, from [37, p. 176, Theorem 14], that, for all $p_{1} \in(0,1]$ and $w \in A_{\infty}\left(\mathbb{R}^{n}\right), K$ is bounded on the weighted Hardy space $H_{w}^{p_{1}}\left(\mathbb{R}^{n}\right)$ and that, for all $s \in(1, \infty), w \in A_{s}\left(\mathbb{R}^{n}\right)$ and $p_{2} \in(2 s, \infty), K$ is bounded on $L_{w}^{p_{2}}\left(\mathbb{R}^{n}\right)$, which, together with Propositions 2.2 and 2.21, and an argument similar to that used in the proofs of Corollaries 2.22 and 2.23, implies that $K$ is bounded on $H_{\varphi}\left(\mathbb{R}^{n}\right)$. This finishes the proof of Proposition 3.6.

Now, let $m \in \mathbb{N}$ and $\mathcal{K}:=\left\{K_{1}, \ldots, K_{m}\right\}$, where, for each $j \in\{1, \ldots, m\}, K_{j}$ is a Fourier multiplier with the multiplier function $\theta_{j} \in C^{\infty}\left(\mathbb{S}^{n-1}\right)$. For any $f \in L^{2}\left(\mathbb{R}^{n}\right)$, let

$$
\mathcal{K}(f):=\left(K_{1}(f), \ldots, K_{m}(f)\right) .
$$

For any $q \in(0, \infty)$, the $q$-order maximal function $\mathcal{M}_{q}(f)$ of $f$ is defined by setting, for all $x \in \mathbb{R}^{n}$,

$$
\mathcal{M}_{q}(f)(x):=\sup _{B \ni x}\left\{\frac{1}{|B|} \int_{B}|f(y)|^{q} d y\right\}^{1 / q},
$$

where the supremum is taking over all balls $B$ of $\mathbb{R}^{n}$ containing $x$. Using Lemma 2.3, we see that, if $i(\varphi)>q q(\varphi), \mathcal{M}_{q}$ is bounded on $L^{\varphi}\left(\mathbb{R}^{n}\right)$.

Now we recall the following result from Uchiyama [38, Theorem 2]. 
Proposition $3.7([38])$. Let $m \in \mathbb{N}, j \in\{1, \ldots, m\}, \theta_{j} \in C^{\infty}\left(\mathbb{S}^{n-1}\right)$ and $\mathcal{K}$, having the form $\left\{K_{1}, \ldots, K_{m}\right\}$, be a vector of Fourier multipliers with the multiplier functions of the form $\left\{\theta_{1}, \ldots, \theta_{m}\right\}$. If

$$
\operatorname{Rank}\left(\begin{array}{ccc}
\theta_{1}(\xi), & \ldots, & \theta_{m}(\xi) \\
\theta_{1}(-\xi), & \ldots, & \theta_{m}(-\xi)
\end{array}\right) \equiv 2
$$

on $\mathbb{S}^{n-1}$, where Rank $(\cdot)$ denotes of the rank of a matrix, then there exist $p_{0} \in(0,1)$ and a positive constant $C$, depending only on $\theta_{1}, \ldots, \theta_{m}$, such that, for all $f \in L^{2}\left(\mathbb{R}^{n}\right)$ and $x \in \mathbb{R}^{n}$,

$$
\mathcal{M}_{\phi}(\mathcal{K}(f))(x) \leq C \mathcal{M}_{p_{0}}\left(\mathcal{M}_{1 / 2}(|\mathcal{K}(f)|)\right)(x),
$$

where $\phi \in \mathcal{S}\left(\mathbb{R}^{n}\right)$ satisfies (2.3),

$$
\mathcal{M}_{\phi}(\mathcal{K}(f)):=\sup _{t \in(0, \infty)}\left|\left(K_{1}(f) * \phi_{t}, \ldots, K_{m}(f) * \phi_{t}\right)\right|,
$$

$|\mathcal{K}(f)|:=\left(\sum_{j=1}^{m}\left|K_{j}(f)\right|^{2}\right)^{1 / 2}, \mathcal{M}_{p_{0}}$ and $\mathcal{M}_{1 / 2}$ are as in (3.5).

Remark 3.8. (i) Inequality (3.7) provides a good substitute for the subharmonic property of $|F|^{p}$ for the harmonic vector (resp. tensor-valued function) $F$, which enables us to use less Riesz transforms than Theorem 1.7 to characterize $H_{\varphi}\left(\mathbb{R}^{n}\right)$, but at the expense that we do not know the exact value of the exponent $p_{0}$ in (3.7).

(ii) Let $k \in \mathbb{N}$ and $\mathcal{K}:=\{I\} \cup\left\{R_{j_{1}} \cdots R_{j_{k}}\right\}_{j_{1}, \ldots, j_{k}=1}^{n}$ consist of the identity operator $I$ and all $k$-order Riesz transforms $R_{j_{1}} \cdots R_{j_{k}}$ defined as in Remark 1.8(i). Then we know that

$$
\operatorname{Rank}\left(\begin{array}{cccc}
1, & \left(-i \xi_{1}\right)^{k}, & \ldots, & \left(-i \xi_{n}\right)^{k} \\
1, & (-1)^{k}\left(-i \xi_{1}\right)^{k}, & \ldots, & (-1)^{k}\left(-i \xi_{n}\right)^{k}
\end{array}\right) \equiv 2
$$

on $\mathbb{S}^{n-1}$ if and only if $k$ is odd. Recall that Gandulfo, García-Cuerva and Taibleson 10 have constructed a counterexample to show that the even order Riesz transforms fail to characterize $H^{1}\left(\mathbb{R}^{2}\right)$. This implies the possibility of using the odd order Riesz transforms to characterize the Hardy type spaces.

Now, we show Theorem 1.11 .

Proof of Theorem 1.11. The proof of the second inequality of (1.12) is an easy consequence of Proposition 2.2 and Corollary 2.22 (see also the proof of the second inequality of (1.10) of Theorem [1.7), the details being omitted.

We now turn to the proof of the first inequality of (1.12). Recall that $\theta_{j_{1}, \ldots, j_{k}}$, defined as in (3.3), is the multiplier function of $R_{j_{1}} \cdots R_{j_{k}}$. From [38, p. 224] (or the proof of [39, p. 170, Theorem 10.2]), we deduce that there exists $\{\psi\} \cup\left\{\psi_{j_{1}, \ldots, j_{k}}\right\}_{j_{1}, \ldots, j_{k}=1}^{n} \subset C^{\infty}\left(\mathbb{S}^{n-1}\right)$ such that, for all $\xi \in \mathbb{S}^{n-1}$,

$$
\psi(\xi)+\sum_{j_{1}, \ldots, j_{k}=1}^{n} \theta_{j_{1}, \ldots, j_{k}}(\xi) \psi_{j_{1}, \ldots, j_{k}}(\xi)=1,
$$


which, together with Proposition [3.6. (3.7), $\frac{i(\varphi)}{q(\varphi)}>\max \left\{p_{0}, \frac{1}{2}\right\}$ and the fact that $\mathcal{M}_{q_{0}} \circ$ $\mathcal{M}_{1 / 2}$ is bounded on $L^{\varphi}\left(\mathbb{R}^{n}\right)$, implies that

$$
\begin{aligned}
\|f\|_{H_{\varphi}\left(\mathbb{R}^{n}\right)} & \leq\left\|(\psi \widehat{f})^{\vee}\right\|_{H_{\varphi}\left(\mathbb{R}^{n}\right)}+\sum_{j_{1}, \ldots, j_{k}=1}^{n}\left\|\left(\theta_{j_{1}, \ldots, j_{k}} \psi_{j_{1}, \ldots, j_{k}} \widehat{f}\right)^{\vee}\right\|_{H_{\varphi}\left(\mathbb{R}^{n}\right)} \\
& \lesssim\|f\|_{H_{\varphi}\left(\mathbb{R}^{n}\right)}+\sum_{j_{1}, \ldots, j_{k}=1}^{n}\left\|R_{j_{1}} \cdots R_{j_{k}}(f)\right\|_{H_{\varphi}\left(\mathbb{R}^{n}\right)} \\
& \lesssim\left\|\mathcal{M}_{\phi}(\mathcal{K}(f))\right\|_{L^{\varphi}\left(\mathbb{R}^{n}\right)} \lesssim\left\|\mathcal{M}_{p_{0}}\left(\mathcal{M}_{1 / 2}(|\mathcal{K}(f)|)\right)\right\|_{L^{\varphi}\left(\mathbb{R}^{n}\right)} \\
& \lesssim\|\mathcal{K}(f) \mid\|_{L^{\varphi}\left(\mathbb{R}^{n}\right)} \lesssim\|f\|_{L^{\varphi}\left(\mathbb{R}^{n}\right)}+\sum_{j_{1}, \ldots, j_{k}=1}^{n}\left\|R_{j_{1}} \cdots R_{j_{k}}(f)\right\|_{L^{\varphi}\left(\mathbb{R}^{n}\right)},
\end{aligned}
$$

where $\mathcal{K}:=\{I\} \cup\left\{R_{j_{1}} \cdots R_{j_{k}}\right\}_{j_{1}, \ldots, j_{k}=1}^{n}, I$ is the identity operator, $\widehat{ }$ and $\vee$ denote, respectively, the Fourier transform and its inverse. This proves the first inequality of (1.12) and hence finishes the proof of Theorem 1.11 .

Acknowledgements. The authors would like to thank the referee for her/his careful reading and several valuable remarks which improve the presentation of this article.

\section{References}

[1] R. A. Adams and J. J. F. Fournier, Sobolev Spaces, Second edition, Elsevier / Academic Press, Amsterdam, 2003, xiv+305 pp.

[2] S. Axler, P. Bourdon and W. Ramey, Harmonic Function Theory, Second edition, Graduate Texts in Mathematics, 137, Springer-Verlag, New York, 2001, xii+259 pp.

[3] A. Bonami, J. Feuto and S. Grellier, Endpoint for the DIV-CURL lemma in Hardy spaces, Publ. Mat. 54 (2010), 341-358.

[4] A. Bonami, S. Grellier and L. D. Ky, Paraproducts and products of functions in $B M O\left(\mathbb{R}^{n}\right)$ and $H^{1}\left(\mathbb{R}^{n}\right)$ through wavelets, J. Math. Pures Appl. (9) 97 (2012), 230241.

[5] H.-Q. Bui, Boundary value characterizations for weighted Hardy spaces of harmonic functions, Forum Math. 2 (1990), 511-521.

[6] A. P. Calderón and A. Zygmund, On higher gradients of harmonic functions, Studia Math. 24 (1964), 211-226.

[7] L. Diening, P. Harjulehto, P. Hästö and M. Ružička, Lebesgue and Sobolev Spaces with Variable Exponents, Lecture Notes in Math. 2017, Springer, Heidelberg, 2011, $\mathrm{x}+509 \mathrm{pp}$.

[8] C. Fefferman, Symposium on Harmonic Analysis, De Paul Univ. Conf., 1974.

[9] C. Fefferman and E. M. Stein, $H^{p}$ spaces of several variables, Acta Math. 129 (1972), 137-195.

[10] A. Gandulfo, J. García-Cuerva and M. Taibleson, Conjugate system characterizations of $H^{1}$ : counter examples for the Euclidean plane and local fields, Bull. Amer. Math. Soc. 82 (1976), 83-85. 
[11] J. García-Cuerva, Weighted $H^{p}$ spaces, Dissertationes Math. (Rozprawy Mat.) 162 (1979), 1-63.

[12] J. García-Cuerva and J. Rubio de Francia, Weighted Norm Inequalities and Related Topics, Amsterdam, North-Holland, 1985, x+604 pp.

[13] L. Grafakos, Classical Fourier Analysis, Second edition, Graduate Texts in Mathematics, 249, Springer, New York, 2008, xvi+489 pp,

[14] L. Grafakos, Modern Fourier Analysis, Second edition, Graduate Texts in Mathematics, 250, Springer, New York, 2009, xvi+504 pp.

[15] S. Hofmann, S. Mayboroda and A. McIntosh, Second order elliptic operators with complex bounded measurable coefficients in $L^{p}$, Sobolev and Hardy spaces, Ann. Sci. École Norm. Sup. (4) 44 (2011), 723-800.

[16] J. Horváth, Sur les fonctions conjuguées à plusieurs variables, Indagationes Math. 15 (1953), 17-29.

[17] S. Hou, D. Yang and S. Yang, Lusin area function and molecular characterizations of Musielak-Orlicz Hardy spaces and their applications, Commun. Contemp. Math. 15 (2013), no. 6, 1350029, 37 pp.

[18] R. Hunt, B. Muckenhoupt and R. Wheeden, Weighted norm inequalities for the conjugate function and Hilbert transform, Trans. Amer. Math. Soc. 176 (1973), 227-251.

[19] S. Janson, Generalizations of Lipschitz spaces and an application to Hardy spaces and bounded mean oscillation, Duke Math. J. 47 (1980), 959-982.

[20] T. Kurokawa, Higher Riesz transforms and derivatives of the Riesz kernels, Integral Transforms Spec. Funct. 15 (2004), 51-71.

[21] L. D. Ky, A note on $H_{w}^{p}$-boundedness of Riesz transforms and $\theta$-Calderón-Zygmund operators through molecular characterization, Anal. Theory Appl. 27 (2011), 251-264.

[22] L. D. Ky, Bilinear decompositions and commutators of singular integral operators, Trans. Amer. Math. Soc. 365 (2013), 2931-2958.

[23] L. D. Ky, New Hardy spaces of Musielak-Orlicz type and boundedness of sublinear operators, Integral Equations Operator Theory 78 (2014), 115-150.

[24] Y. Liang, J. Huang and D. Yang, New real-variable characterizations of Hardy spaces of Musielak-Orlicz type, J. Math. Anal. Appl. 395 (2012), 413-428.

[25] B. Muckenhoupt, Weighted norm inequalities for the Hardy maximal function, Trans. Amer. Math. Soc. 165 (1972), 207-226.

[26] B. Muckenhoupt and R. L. Wheeden, On the dual of weighted $H^{1}$ of the half-space, Studia Math. 63 (1978), 57-79.

[27] J. Musielak, Orlicz Spaces and Modular Spaces, Lecture Notes in Math. 1034, Springer-Verlag, Berlin, 1983, iii +222 pp.

[28] S. Nualtaranee, On least harmonic majorants in half-spaces, Proc. London Math. Soc. (3) 27 (1973), 243-260.

[29] M. Peloso and S. Secco, Local Riesz transforms characterization of local Hardy spaces, Collect. Math. 59 (2008), 299-320.

[30] E. M. Stein, On the theory of harmonic functions of several variables. II. Behavior near the boundary, Acta Math. 106 (1961), 137-174.

[31] E. M. Stein, Singular Integrals and Differentiability Properties of Functions, Princeton University Press, Princeton, N. J., 1970, xiv+290 pp. 
[32] E. M. Stein, Harmonic Analysis: Real-variable Methods, Orthogonality, and Oscillatory Integrals, Princeton University Press, Princeton, N. J., 1993, xiv+695 pp.

[33] E. M. Stein and G. Weiss, On the theory of harmonic functions of several variables. I. The theory of $H^{p}$-spaces, Acta Math. 103 (1960), 25-62.

[34] E. M. Stein and G. Weiss, Generalization of the Cauchy-Riemann equations and representations of the rotation group, Amer. J. Math. 90 (1968), 163-196.

[35] E. M. Stein and G. Weiss, Introduction to Fourier Analysis on Euclidean Spaces, Princeton University Press, Princeton, N. J., 1971, x+297 pp.

[36] J.-O. Strömberg, Bounded mean oscillation with Orlicz norms and duality of Hardy spaces, Indiana Univ. Math. J. 28 (1979), 511-544.

[37] J.-O. Strömberg and A. Torchinsky, Weighted Hardy Spaces, Lecture Notes in Math. 1381, Springer-Verlag, Berlin, 1989, vi+193 pp.

[38] A. Uchiyama, The Fefferman-Stein decomposition of smooth functions and its application to $H^{p}\left(\mathbb{R}^{n}\right)$, Pacific J. Math. 115 (1984), 217-255.

[39] A. Uchiyama, Hardy Spaces on the Euclidean Space, Springer-Verlag, Tokyo, 2001, xiv +305 pp.

[40] B. Viviani, An atomic decomposition of the predual of $B M O(\rho)$, Rev. Mat. Iberoamericana 3 (1987), 401-425.

[41] R. L. Wheeden, A boundary value characterization of weighted $H^{1}$, Enseignement Math. (2) 22 (1976), 121-134.

[42] R. L. Wheeden, On the dual of weighted $H^{1}(z<1)$, Approximation theory (Papers, VIth Semester, Stefan Banach Internat. Math. Center, Warsaw, 1975), pp. 293-303, Banach Center Publ., 4, PWN, Warsaw, 1979.

[43] K. Yabuta, Generalizations of Calderón-Zygmund operators, Studia Math. 82 (1985), 17-31.

[44] D. Yang and S. Yang, Musielak-Orlicz Hardy spaces associated with operators and their applications, J. Geom. Anal. 24 (2014), 495-570.

Jun Cao and Dachun Yang (Corresponding author)

School of Mathematical Sciences, Beijing Normal University, Laboratory of Mathematics and Complex Systems, Ministry of Education, Beijing 100875, People's Republic of China E-mails: caojun1860@mail.bnu.edu.cn (J. Cao) dcyang@bnu.edu.cn (D. Yang)

Der-Chen Chang

Department of Mathematics and Department of Computer Science, Georgetown University, Washington D.C. 20057, USA

Department of Mathematics, Fu Jen Catholic University, Taipei 242, Taiwan

E-mail: chang@georgetown.edu

Sibei Yang

School of Mathematics and Statistics, Lanzhou University, Lanzhou, Gansu 730000, People's Republic of China

E-mails: yangsb@lzu.edu.cn 